\title{
The recent star-formation history of the Large and Small Magellanic Clouds
}

\author{
G. Indu ${ }^{1,2}$ and A. Subramaniam ${ }^{1}$ \\ ${ }^{1}$ Indian Institute of Astrophysics, Koramangala II Block, Bangalore-560034, India \\ e-mail: [indu;purni]@iiap.res.in \\ ${ }^{2}$ Pondicherry University, R. Venkataraman Nagar, Kalapet, Pondicherry-605014, India
}

Received 20 May 2011 / Accepted 31 August 2011

\begin{abstract}
Aims. Recent interactions between the Large and the Small Magellanic Clouds (LMC and SMC) and the Milky Way can be understood by studying their recent star formation history. This study aims to detect any directional or propagating star formation in the last 500 Myr.

Methods. We traced the age of the last star-formation event (LSFE) in the inner Large and Small Magellanic Cloud (L\&SMC) using the photometric data in $V$ and $I$ passbands from the Optical Gravitational Lensing Experiment (OGLE-III) and the Magellanic Cloud Photometric Survey (MCPS). The LSFE is estimated from the main sequence turn-off point in the color-magnitude diagram (CMD) of a subregion. After correcting for extinction, the turn-off magnitude is converted to age, which represents the LSFE in a region.

Results. The spatial distribution of the age of the LSFE shows that the star-formation has shrunk to within the central regions in the last $100 \mathrm{Myr}$ in both the galaxies. The location as well as age of LSFE is found to correlate well with those of the star cluster in both the Clouds. The SMC map shows two separate concentrations of young star-formation, one near the center and the other near the wing. We detect peaks of star-formation at 0-10 Myr and 90-100 Myr in the LMC, and 0-10 Myr and 50-60 Myr in the SMC. The quenching of star-formation in the LMC is found to be asymmetric with respect to the optical center such that most of the young star forming regions are located to the north and east. On deprojecting the data onto the LMC plane, the recent star-formation appears to be stretched in the northeast direction and the HI gas is found to be distributed preferentially in the north. We found that the centroid is shifted to the north during the time interval 200-40 Myr, whereas it is found to have shifted to the northeast in the last 40 Myr. In the SMC, we detect a shift in the centroid of the population younger than $500 \mathrm{Myr}$ and as young as $40 \mathrm{Myr}$ in the direction of the LMC.

Conclusions. We propose that the HI gas in the LMC has been pulled to the north of the LMC in the last 200 Myr because of the gravitational attraction of our Galaxy at the time of perigalactic passage. The shifted HI gas was preferentially compressed in the north during the time interval 200-40 Myr and in the northeast in the last $40 \mathrm{Myr}$, owing to the motion of the LMC in the Galactic halo. The recent star-formation in the SMC is due to the combined gravitational effect of the LMC and the perigalactic passage.
\end{abstract}

Key words. stars: formation - galaxies: kinematics and dynamics - galaxies: evolution - Magellanic Clouds - galaxies: star formation

\section{Introduction}

The Large and Small Magellanic Clouds (L\&SMC), along with the components of the bridge and the stream, comprise the Magellanic system. The presence of the bridge and the stream connecting the two Clouds suggest that these two galaxies might have been together possibly as an interacting pair. The Bridge in particular indicates that they have had a close encounter in the recent past. This system moves in the gravitational potential of the Galaxy. It is obvious that the structure, kinematics, and evolution of the clouds and the Galaxy are modified by their interactions. The Magellanic Clouds are gas rich and have active ongoing star-formation, possibly triggered by interactions between the Magellanic Clouds (MCs) and interactions of the clouds with the Galaxy. It was long believed that the Clouds orbit our Galaxy and that the bursts of star-formation episodes seen in both the Clouds are probably due to their perigalactic passage and tidal effects (Harris \& Zaritsky 2004; Lin et al. 1995). On the other hand, the recent estimates of the proper motion of the Clouds find that the Magellanic System is probably passing close to the Milky Way (MW) for the first time (Besla \& Kallivayalil 2007). Thus, the star-formation episodes which were attributed to the perigalactic passage need to be reconsidered. Nevertheless, the star-formation history (SFH) of the MCs have been studied to identify the interaction between the Clouds and their ages, as mentioned below, assuming that an interaction induces simultaneous star-formation in both the galaxies. In addition, the starformation induced by an interaction can lead to star-formation propagating within the galaxies and the direction of propagation could provide valuable clues about the details of interaction. Since the Clouds are presently passing near the Galaxy and are together, the pattern of the recent star-formation is likely to indicate the effects of the Galaxy-LMC-SMC interaction. In this work, we study the pattern of the recent star-formation in the Clouds, with specific interest in tracing the origin and nature of the interaction that caused it.

The recent SFH has been studied by various authors using star clusters as well as the field star population. The star clusters in the LMC were studied and their derived age distribution compared with that in the SMC by Pietrzynski \& Udalski (2000). The comparison of both cluster formation and star-formation is also done to find the correlation between the two processes (Holtzman et al. 1999; Subramaniam 2004). Harris \& Zaritsky (2009, hereafter H\&Z09) reconstructed the SFH of the LMC and 
concluded that field and cluster star-formation modes are tightly coupled. They found a quiescent epoch from 12 to 5 Gyr ago and star-formation peaks at $2 \mathrm{Gyr}, 500 \mathrm{Myr}, 100 \mathrm{Myr}$, and $12 \mathrm{Myr}$. The study of the spatial distribution of clusters as well as starformation rates are also equally interesting. A study of the distribution of the bar cluster population in the LMC (Bica et al. 1992) has shown that clusters younger than 200 Myr are not homogeneously distributed throughout the bar. In particular, a strong star-formation event at $100 \mathrm{Myr}$ was detected in the eastern part of the bar. Harris \& Zaritsky (2004) studied the SFH of the SMC and found a quiescent epoch between 8.4 and 3 Gyr. They also found evidence of a continuous star-formation from 3 Gyr to the present epoch with star-formation peaks at 2-3 Gyr, 400 and 60 Myr. Noel et al. (2009) also studied the SFH of the SMC and found that the younger stars (200-500 Myrs) have an asymmetric distribution with the appearance of the wing, while the older population $(>1 \mathrm{Gyr})$ is distributed similarly at all radii and all azimuths. In contrast to Harris \& Zaritsky (2004), Noel et al. (2009) did not find any quiescent epoch at the intermediate ages. Glatt et al. (2010) studied the SFH of both the clouds based on star clusters with age $<1$ Gyr. They found that the cluster formation peaks at $160 \mathrm{Myr}$ and $630 \mathrm{Myr}$ for the SMC and $125 \mathrm{Myr}$ and $800 \mathrm{Myr}$ for the LMC. Thus, the studies completed so far have found that the age of the star-formation peaks in the LMC and the SMC fall in the similar range, but the values do not coincide. Using MACHO Cepheids as tracers, Alcock et al. (1999) found that the star-formation in the LMC has propagated from southeast to northwest, along the bar, in the last $100 \mathrm{Myr}$. Though some studies have found that there is evidence of propagating star-formation, the details are not clear. Study of a larger area using homogeneous data is required to obtain the details of any propagating star-formation.

There are different models for the evolution of star-formation in irregular, low-mass spiral galaxies that depict the spatial variation in star-formation. Gallart et al. (2008) presented an outsidein disk evolution in the LMC, which is explained in terms of decreasing HI column density with galactocentric distance. They observed an outside-to-inside quenching of star-formation, such that, a field at $2.3^{\circ}$ is currently active in star-formation, while fields at $4.4^{\circ}, 5.5^{\circ}$, and $7.1^{\circ}$ have $100 \mathrm{Myr}, 0.8 \mathrm{Gyr}$, and $1.5 \mathrm{Gyr}$ old stars as the youngest population, respectively. Thus, the age of the youngest stars in each field gradually increases with distance from the center and the population is found to be older on average towards the outer part of the galaxy (Saha et al. 2010; Gallart et al. 2009). It will be interesting to ascertain out to which radius this outside-in quenching of star-formation can be traced. This study also requires homogeneous data over a large spatial area of the two Clouds.

The most common method used to constrain the SFH involves a quantitative comparison of observed colour-magnitude diagrams (CMD) with synthetic CMDs constructed using theoretical isochrones according to an adopted IMF and input model SFH. H\&Z09 performed such a study for the LMC using the MCPS data, where they modelled the complete SFH of the LMC. They used just five time steps to represent the recent SFH (6.3 Myr, 12.5 Myr, 25 Myr, 50 Myr, and $100 \mathrm{Myr}$ ), which is equal to 0.3 in log (age). The gaps between the steps are large and these large gaps in the recent SFH provide insufficient time resolution to identify and study any propagating star-formation. If one aims to study the recent SFH with a time resolution of 5-10 Myr for ages younger than $100 \mathrm{Myr}$, then it is more efficient to model only the young stars and not the entire range of stars in a given region. Since large numbers of stars are required in the CMD of a region to model the full range of age, the area required is also large and the spatial resolution is consequently low. To achieve a higher spatial as well as temporal resolution, we adopt a different method. In this method, we estimated the age of the last star-formation event (LSFE) in a given region, by identifying the turn-off of the main sequence (MS) in the CMD of the corresponding region. The turn-off identified from the luminosity function (LF) of the MS represents the last star-formation event experienced by the region. The reddening in the direction of this region is also estimated from the turn-off. The spatial map of age of the LSFE is used to help us identify any propagating star-formation. We also produce a map of the average reddening in regions studied in both the galaxies. The spatial and temporal resolutions achieved using this method are both higher than those obtained using the traditional method, as only a small range in age is studied. For the same reason, this method does not assume any age-metallicity relation.

The paper is organised as follows. Section 2 describes the data and Sect. 3 outlines our methodology. The results are presented in Sect. 4, with subsections for extinction and LSFE maps of the LMC and the SMC. Discussion is presented in Sect. 5. The error estimates are presented in Appendix A.

\section{Data}

This study makes use of two publicly available photometric survey data that cover large areas of the MCs. These are the catalogs produced by the Optical Gravitational Lensing Experiment (OGLE-III, Udalski et al. 2008a,b) and the Magellanic Cloud Photometric Survey (MCPS, Zaritsky et al. 2002, 2004). The OGLE III photometric maps form a significant extension of OGLE II. The total observed area is 40 square degrees, covering 116 LMC regions, each of which covers an area of $35 \times 35 \operatorname{arcmin}^{2}$. The catalog consists of calibrated photometry in $V$ and $I$ passbands of about 35 million stars. Each of the 116 regions are observed using eight chips each with an area of $8.87 \times 17.74 \mathrm{arcmin}^{2}$. In this study, each of these were divided into subregions of three different areas of $4.43 \times 4.43$, $4.43 \times 8.87$, and $8.87 \times 8.87 \mathrm{arcmin}^{2}$. The different sizes for the subregions are chosen, to study how their area, effects the identified turn-off magnitude and thus the estimated age of the LSFE. For the SMC the catalog consists of $V$ and $I$ photometry of 6.5 million stars from 41 fields, covering an area of 14 square deg in the sky. For this study the area binning in the SMC was done with $4.43 \times 4.43,4.43 \times 8.87$, and $8.87 \times 8.87 \mathrm{arcmin}^{2}$.

The MCPS spans a total area of 64 square deg, and contains about 24 million objects in the LMC. The survey presents photometric as well as extinction maps in $U, B, V$, and $I$ passbands. In the case of the MCPS, the subregions have area in the range, $10.5 \times 30 \operatorname{arcmin}^{2}, 10.5 \times 15 \operatorname{arcmin}^{2}$, and $5.3 \times 15 \operatorname{arcmin}^{2}$. The MCPS survey consists of UBVI photometry of the central 18 square deg of the SMC covering 5 million stars. Subregions of area $10.5 \times 30 \mathrm{arcmin}^{2}, 10.5 \times 15 \mathrm{arcmin}^{2}$, and $5.3 \times 15 \mathrm{arcmin}^{2}$ were made to estimate the age of the LSFE. For uniformity, we use the $V$ and $I$ photometric data from both the catalogs.

\section{Methodology}

\subsection{Identifying the MS turn-off}

We adopted the following method to identify the age of the LSFE from the CMDs. The observed region is divided into several smaller subregions to increase the spatial resolution. The area of the smallest subregion is decided based on the number of MS stars in the CMD, that is, it has a minimum of about 250 MS stars in the SMC 600 MS stars in the LMC. For each subregion, $(V-I)$ versus (vs.) $V \mathrm{CMD}$ is constructed and the 
MS is identified as stars brighter than 21 mag and with a color index less than $0.5 \mathrm{mag}$. The turn-off is identified from the MS by constructing the LF which involves binning in $V$ magnitude with a bin size of $0.2 \mathrm{mag}$. The brightest bin in the LF is identified using a statistical cut-off of $2 \sigma$ significance (minimum five stars in the brightest bin). The cut-off means that the tip of the MS should have at least five stars such that it has a variance of $\sqrt{(5)}=2.2$. Thus, the number of stars identified as the tip of the MS is more than twice the variance. This cut-off is chosen to reduce the statistical fluctuation in identifying the MS turnoff. Thus, after computing the LF of the MS, the brightest bin that has five or more stars is identified as the tip of the MS. This condition also implies that, the age of the LSFE identified in a subregion will have a certain threshold star-formation rate to form so many stars. On the other hand, star-formation events with rates lower than this threshold will not be identified. The above condition would also minimise the chances of identifying blue supergiants as MS stars. Since the number of stars in the brightest bin depends on the area used, the age of the LSFE will also depend on the area of the bin considered. Thus we used three sizes to describe the area of the subregions, to map the age of the LSFE. The average $V$ magnitude corresponding to the brightest bin with the required number of stars is taken as the tip of the MS. This is considered as the turn-off magnitude $V_{\text {to }}$ of the youngest stellar population present in the region.

To convert $V_{\text {to }}$ to age, it has to be corrected for extinction, which we estimated from the colour of the turn-off. This colour of the turn-off was in turn identified as the densest point on the MS, which appears as a peak in a colour distribution of the stars near the turn-off. In other words, the peak of the distribution of stars with respect to the $(V-I)$ color near the turn-off can be used to identify the colour of the MS. To estimate the peak $(V-I)$ color of the turn-off, a strip parallel to $(V-I)$ axis with a width $0.5 \mathrm{mag}$ is considered (given by $V_{\text {to }}+0.5 \mathrm{mag}$ ). This strip is binned in colour (with a bin size of $0.1 \mathrm{mag}$ ) to study the distribution of stars along the $(V-I)$ colour. This distribution is found to have a unique peak and asymmetric wings. The $(V-I)$ bin corresponding to the peak of the distribution is identified as the location of the MS. The average value of the bin corresponding to this peak was taken as the $(V-I)$ colour of the MS turn-off. Since we consider the peak of the distribution and not the average, the colour estimated corresponds only to the MS stars. As the distribution is found to be asymmetric, the average is likely to be redder than the peak. The bin sizes used to estimate the turn-off magnitude and colour are same for both the LMC and the SMC. Since we statistically trace the brightest part of the CMD in a region, this method is not significantly affected by crowding or incompleteness of the data. The following section describes the method that we used to estimate the reddening and extinction.

\subsection{Estimation of reddening and turn-off age}

We estimated the reddening towards a subregion, from the colour of the turn-off identified from the CMD. The reddening is estimated as the difference between the estimated colour of the turn-off and the expected colour. Hence one needs to know the expected $M_{v}$ and the $(V-I)_{0}$. Since we know the distance modulus (DM), by applying an average value of extinction to the observed apparent magnitude we can approximately estimate the $M_{v}$, given by

$M_{v}=m_{v}-D M-A_{v}$
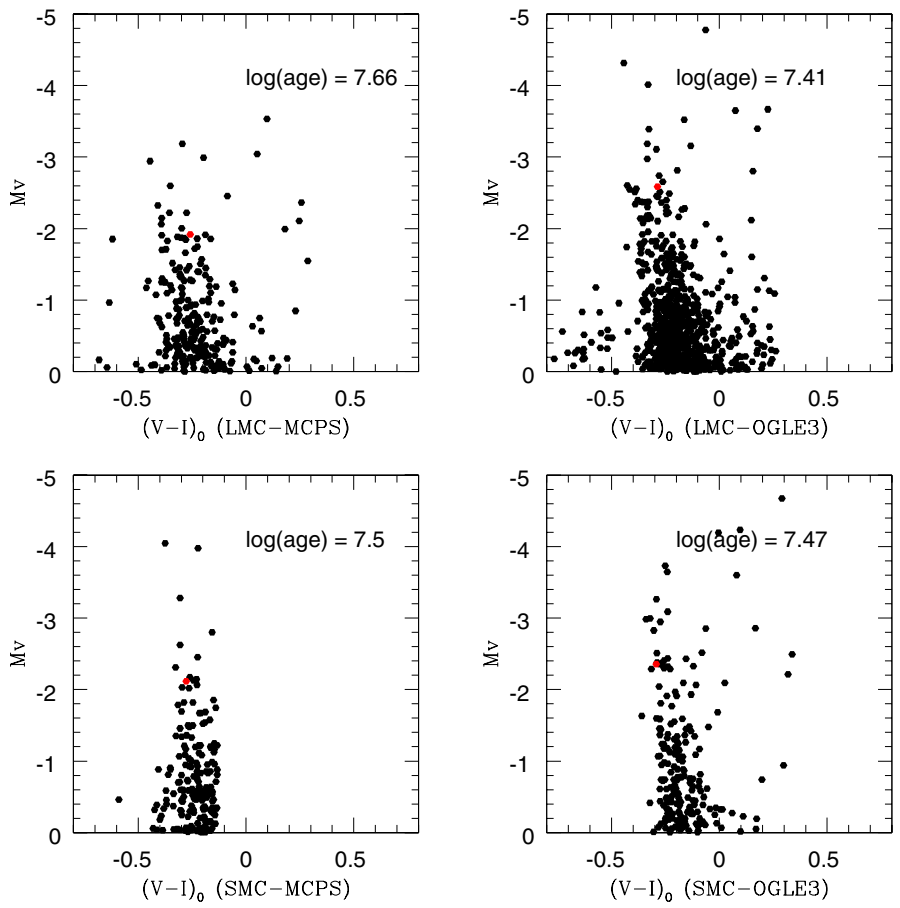

Fig. 1. $M_{v}$ vs. $(V-I)_{0}$ CMDs for two regions each in the L\&SMC. The top panels show two regions in the LMC, with left panel using MCPS data and the right using OGLE III data. Similarly, bottom panels show two regions in the SMC (left using MCPS and right using OGLE III). The red dot marks the turn-off point, and the estimated turn-off age is also shown.

where $m_{v}$ is the apparent magnitude. An initial extinction value is needed to calculate the $M_{v}$. To begin with, we assumed an extinction of $A_{v}=0.55$ (Zaritsky et al. 2004), along with a distance modulus of 18.5, for the LMC. This value of $A_{v}$ is the average value of the extinction towards the LMC. $M_{v}$ thus obtained is used to identify the approximate location of the turn-off and its expected color $(V-I)_{0}$ from the isochrones of Marigo et al. (2008) for a metallicity of $Z=0.008$. The difference between the expected colour and the observed colour of the turn-off is defined as the colour excess for a subregion

$E(V-I)=(V-I)-(V-I)_{0}$.

Then the actual value of $A_{v}$ for each subregion is found using the formula (Nikolaev et al. 2004)

$A_{v}=2.48 E(V-I)$.

Thus the reddening, $E(V-I)$, and extinction, $A_{v}$ are estimated for the LMC subregions. In the case of the SMC, a constant distance modulus of 18.9 and an average extinction value of $A_{v}=0.46 \mathrm{mag}$ (Zaritsky et al. 2002) are used. The expected value of the unreddened colour, $(V-I)_{0}$ is obtained from Marigo et al. (2008) isochrones for a metallicity of $Z=0.004$. The above equations are used to estimate the extinction towards each subregion. Using these estimated values of extinction, the actual magnitude $M_{v}$ corresponding to the turn-off is estimated for all the subregions. Applying the calculated colour excess and extinction for each subregion, CMDs can be obtained with absolute magnitude, $M_{v}$, and dereddened color, $(V-I)_{0}$. The CMDs of some sample regions are shown in Fig. 1. The upper panels show the CMDs of the LMC subregions (MCPS on the left and OGLE III on the right), whereas the lower panels show the CMDs of the SMC subregions. The red dot on the MS shows the location of 

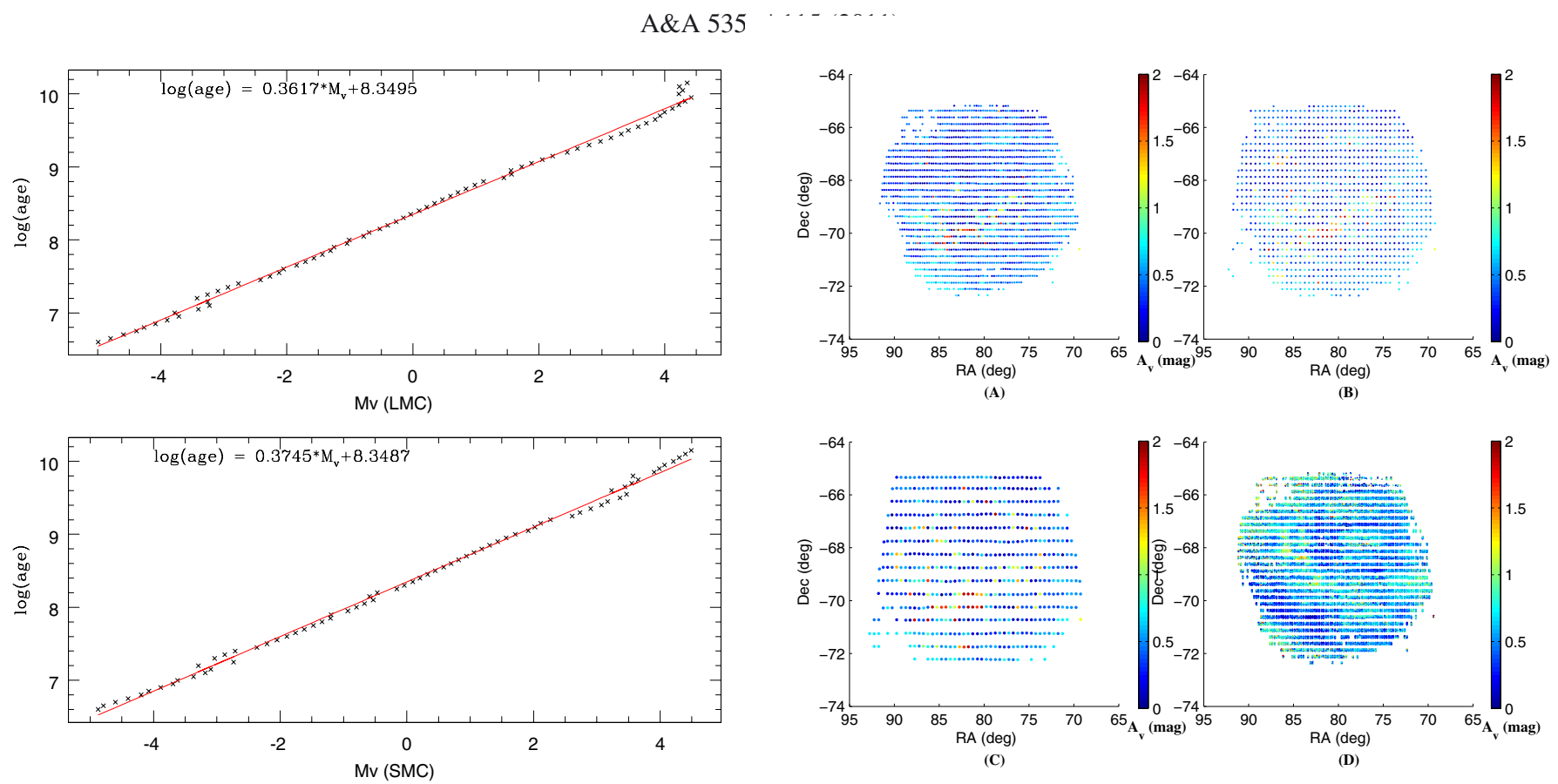

Fig. 2. The log (age) vs. $M_{v}$ plots for L\&SMC, in the left and right panels, respectively. The turn-off $M_{v}$ corresponding to various ages are inferred from Marigo et al. (2008) isochrones. The fitted line is shown in red in both the plots.

the identified turn-off. We show various cases here, where we can see broad/tight MS turn-offs. For example, we consider the region shown in the top right panel, which corresponds to the location with mean $\mathrm{RA}=79.8^{\circ}$ and $\mathrm{Dec}=-69.3^{\circ}$. It covers an area of $4.43 \times 8.87 \mathrm{arcmin}^{2}$. The total number of stars is about 6090 . The identified apparent turn-off magnitude is 16.5 , and the color index is -0.05 . Applying the initial extinction value and DM as described above, the approximate location of the MS is identified to have an absolute magnitude of $16.5-18.5-0.55=-2.55$. The nearest absolute $M_{v}$ value from the isochrone table is -2.54 . The corresponding $(V-I)_{0}$ value is -0.287 . Thus, the reddening towards this region is estimated as $E(V-I)=0.237$ and a corresponding extinction, $A_{v}=0.588 \mathrm{mag}$. Correcting the turn-off $m_{v}$ for extinction using these values, we estimate the MS turn-off at $M_{v}=-2.588 \mathrm{mag}$. Converting this turn-off magnitude into an age with the estimated conversion relation, we get $\log ($ age $)=7.41$.

The estimated turn-off $M_{v}$ is used to estimate the age of the LSFE in each subregion. We estimated an age- $M_{v}$ relation for the turn-off, using the isochrones of Marigo et al. (2008). The age- $M_{v}$ relation is obtained for both the LMC and the SMC separately, where the metallicity of the isochrones is chosen to be 0.008 for the LMC and 0.004 for the SMC. The plots of the $\log$ (age) vs. $M_{v}$ for the L\&SMC are given in Fig. 2 . The relation is found to be linear and we have derived a linear relation between the two by fitting a line to the data points. The turnoff ages for the sample regions, estimated using the above relation are also shown in Fig. 1. The identification of MS turn-off was found to be ambiguous for turn-offs fainter than 18.0 mag. Therefore, we have put a limiting apparent turn-off magnitude of 18.0 , for both the L\&SMC, which will eventually lead to a higher cut-off for the age of the LSFE, around $120 \mathrm{Myr}$ for the LMC and $100 \mathrm{Myr}$ for the SMC. Therefore, subregions with turn-off magnitudes fainter than 18.0 mag are not considered and these locations will appear as gaps in the extinction and LSFE maps.

Since the number of stars in the CMD increases with the area of the subregion considered, the derived turn-off parameters,

Fig. 3. The extinction map of the LMC using MCPS data in the RADec plane with three different area binning, A. $5.3 \times 15 \operatorname{arcmin}^{2}$, B. $10.5 \times 15 \mathrm{arcmin}^{2}$, and C. $10.5 \times 30 \mathrm{arcmin}^{2}$. The bottom right panel is the extinction map of Zaritsky et al. (2004), where the regions used in our analysis are selected and shown. Color coding is according to the $A_{v}$ value, which varies from 0.2 to 2.0 as shown in the color bar.

extinction, and the age of the LSFE will depend on the area considered. To determine the effect of area on the estimated parameters, we derived the extinction and the age of the LSFE for all the three sizes of subregions, in both the data sets and in both the galaxies. We estimated the errors in the estimation of extinction and age using two methods. We simulated synthetic CMDs using Marigo et al. (2008) isochrones for the LMC and the SMC. The synthetic CMDs were analysed similarly to the observed CMDs to estimate the errors as a function of the turn-off magnitude, colour, extinction, and sampling. The errors are also estimated using the method of propagation of errors. These are described in Appendix A.

\section{Results}

\subsection{LMC: extinction}

For all the regions, the estimated colour excess $E(V-I)$ and extinction $A_{v}$ can be used to create an extinction map of the LMC. The extinction is estimated from the brightest MS stars in each region. Figure 3 shows the map estimated using the MCPS data, and Fig. 4 shows the map estimated using the OGLE III data. Each figure has three panels which show the extinction estimated using area bins as indicated in the figure. Since the estimated extinction would depend on the area used, the corresponding maps are used for the estimation of age of the LSFE.

The extinction map estimated from the MCPS data (Fig. 3) shows that the extinction varies within the range $A_{V}=0.2-$ 2.0 mag. Relatively high extinction is seen in the bar region, with the eastern part having higher extinction. A few regions in the north eastern part are also found to have high extinction. These features are seen in all the three plots, which show extinction for different area bins. It can be seen that with the increase in area, the estimated extinction increases. The average value increases from about 0.4 to $0.6 \mathrm{mag}$, from the maps A to $\mathrm{C}$. The extinction map presented in Fig. 4 is obtained from OGLE III data and has 


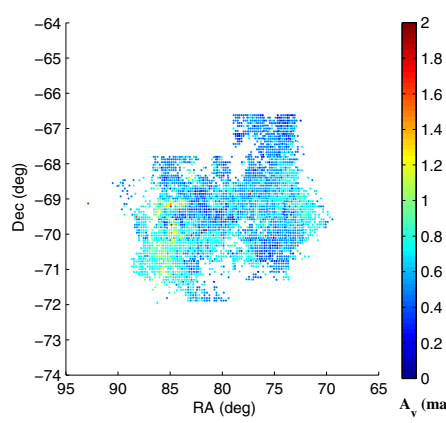

(A)

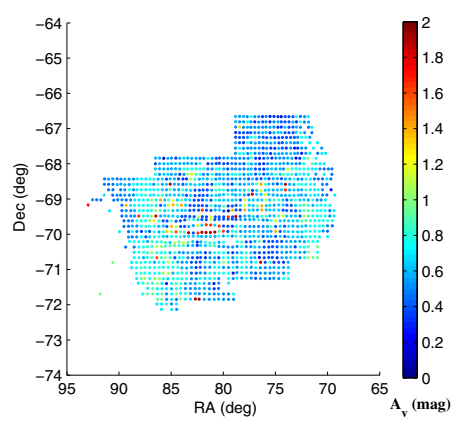

(C)

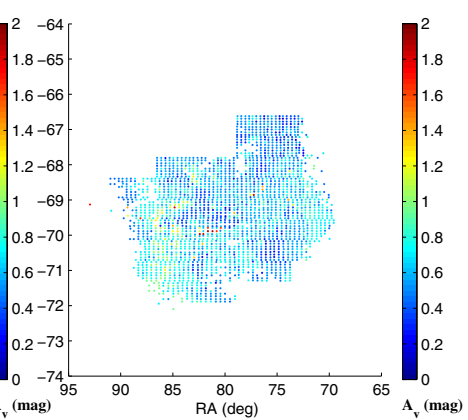

(B)

A map - LMC (OGLE III)

Bin size used :

A) $4.4 \times 4.4$ arcmin $^{2}$

B) $4.4 \times 8.8 \mathrm{arcmin}^{2}$

C) $8.8 \times 8.8 \operatorname{arcmin}^{2}$
Fig. 4. The extinction map of the LMC, similar to Fig. 3, using OGLE III data.

smaller area coverage and higher resolution. The extinction estimated here is also in the range $A_{V}=0.2-2.0$ mag. These maps show that the bar region has the highest extinction, along with the 30 Doradus region. The northern star forming regions are not covered here. The eastern region is found to have high extinction and this region coincides with the location of massive HI clouds, extending out to the 30 Doradus star forming region. The map A shows that the extinction has a very clumpy distribution with pockets of lower extinction. We also note the increase in extinction with the increase in area in these maps. These maps will be useful for studies related to young stars, since the reddening in the LMC is known to depend on the population studied (Zaritsky et al. 2004).

Zaritsky et al. (2004) published a reddening map of the early-type stars in the LMC derived from the MCPS data using stars with effective temperature from 12000 to $45000 \mathrm{~K}$. They presented individual reddenings to stars and hence can be considered as a high resolution map. We compare the reddening distribution derived in this paper with that of Zaritsky et al. (2004). The histograms in Fig. 5 shows the distribution of our extinction values (shown as solid line) and the extinction map provided by Zaritsky et al. (2004) (shown as dashed line). We used the $10.5 \times 15 \operatorname{arcmin}^{2}$ area bin for comparison. The distributions are found to be more or less similar. The peaks of both the distributions coincide at $A_{v}=0.5 \mathrm{mag}$. The reddening estimated here in this study has more regions with less than the peak value. This may be because that we estimate extinction for stars located on the MS and ignore stars that are redder than the MS. Since we use a bin size of 0.2 mag in colour, the mean of this bin is taken as the colour of the MS and hence could introduce a shift of $\pm 0.1 \mathrm{mag}$ in reddening. This would correspond to a shift of 0.25 in extinction. The variation one notices between the two distributions, as seen in Fig. 5, is of this order. This variation in the extinction measurement can introduce a shift in the estimated age which we account for estimating the error in age estimation (see Appendix A). In Fig. 3D, we show the extinction

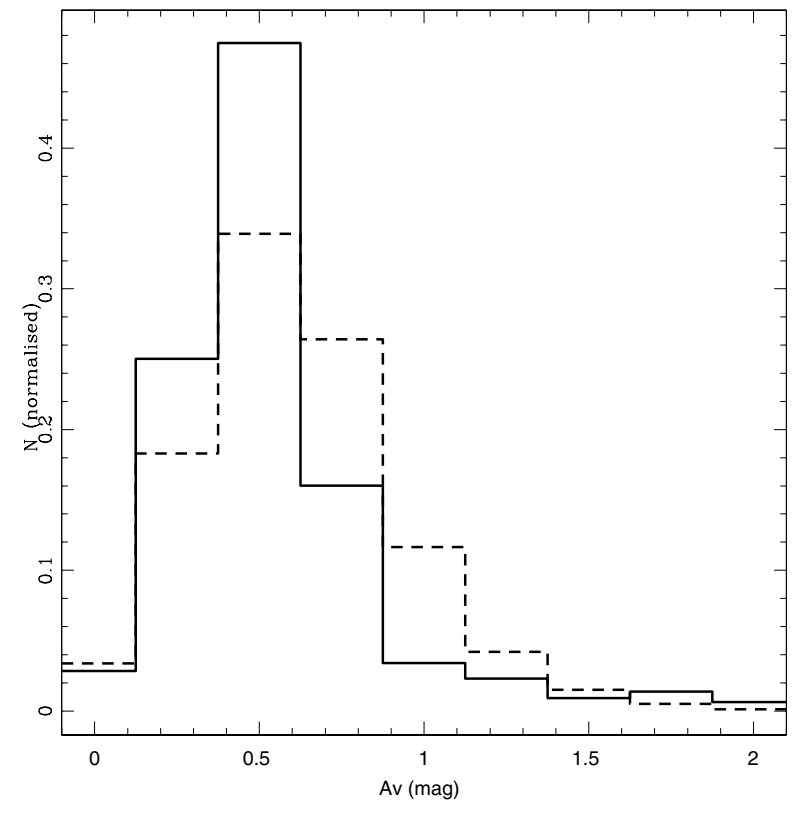

Fig. 5. The estimated distribution of extinction in the LMC (shown as solid line) is compared with distribution obtained from the extinction map of hot stars provided by Zaritsky et al. (2004) (shown as dashed line, extracted from the fits image from the authors' website).

map estimated by Zaritsky et al. (2004) for the regions analysed in this study. This map was obtained by spatially correlating the Zaritsky et al. (2004) map with our sample of selected regions in the MCPS data. The map includes extinction estimates of all the stars present in a given region. This map can be compared with the MCPS extinction maps derived in this study (shown in Figs. $3 \mathrm{a}-\mathrm{c}$ ). The maps are more or less comparable. We do not detect isolated high extinction values, probably because we derive extinction values for regions and not individual stars.

\subsection{LMC: distribution of age of the LSFE}

We estimated the age of the LSFE across the LMC for subregions of three different sizes. The distribution of age of the LSFE can be used to study the quenching as well as the propagation of star-formation in the central regions of the LMC. The maps of the age of the LSFE derived from the MCPS and the OGLE III data are shown in Figs. 6 and 7, respectively.

Figure 6 shows that the age of the LSFE is within the range 0-120 Myr. The figure shows maps for three different area bins as indicated. When we inspect the high resolution map A, we can see that it is a clumpy distribution with many central pockets of very young age, as suggested by the dark blue colour points. The youngest ages are found in the bar region, near the 30 Doradus and the northern regions. The locations are similar to the regions identified by H\&Z09, such as the blue arm, constellation III, and 30 Doradus. These young pockets are surrounded by regions of older star-formation. We can also see that the age of the LSFE is found to progressively increase as we go towards the outer regions. We also see a couple of small pockets of older star-formation in the inner regions. The map also identifies the northwestern void. On the whole, the inner regions have ages of the range 0-40 Myr, whereas regions towards the periphery have ages in the range 60-100 Myr. The age map for larger area bins are shown in B and $\mathrm{C}$. These maps identifies lesser details, when compared to the map A. The clumps seen in map A disappear in maps B and C. As mentioned earlier, the age limit of 


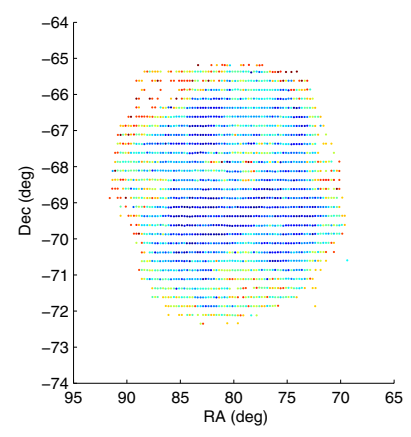

(A)

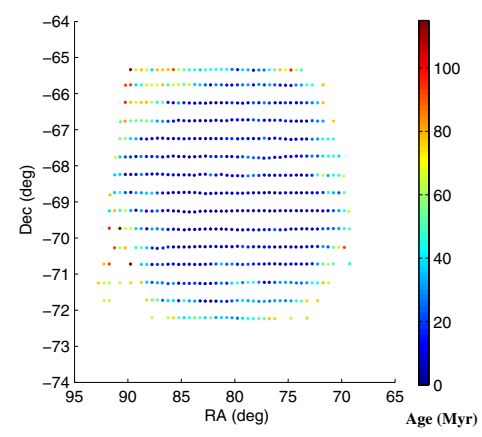

(C)

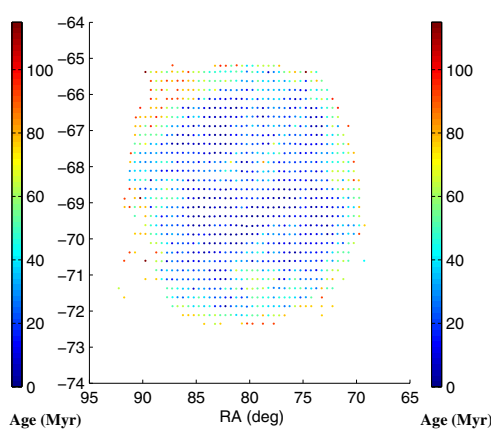

(B)

LSFE map - LMC (MCPS)

Bin size used :

A) $5.3 \times 15 \mathrm{arcmin}^{2}$

B) $10.5 \times 15 \operatorname{arcmin}^{2}$

C) $10.5 \times 30 \operatorname{arcmin}^{2}$
Fig. 6. The LSFE map of the LMC using MCPS data, in the RA-Dec plane with three different area binning, as specified in the figure. Color coding is according to the LSFE age as shown in the color bar.

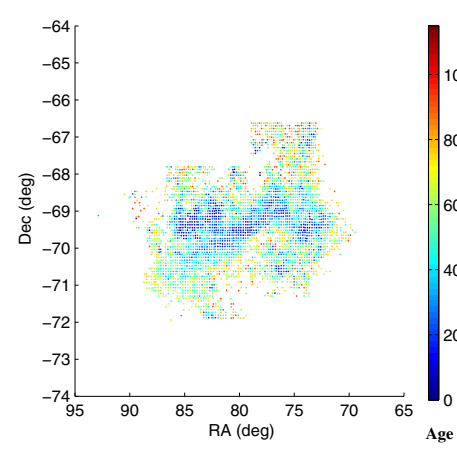

(A)

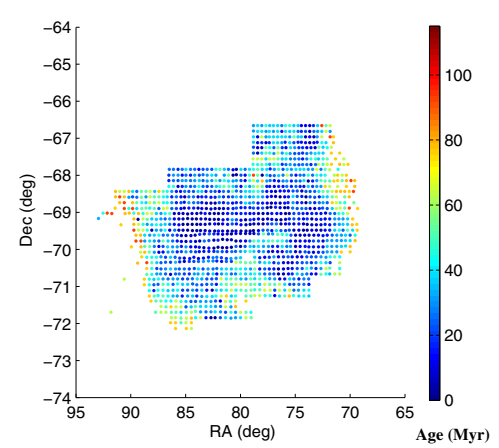

(C)

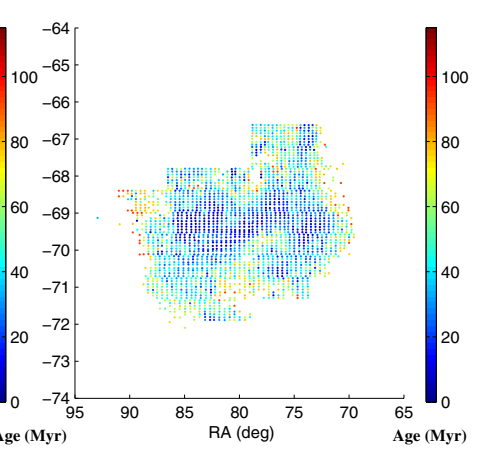

(B)

LSFE map - LMC (OGLE III)

Bin size used :

A) $4.4 \times 4.4 \operatorname{arcmin}^{2}$

B) $4.4 \times 8.8 \operatorname{arcmin}^{2}$

C) $8.8 \times 8.8 \operatorname{arcmin}^{2}$
Fig. 7. The LSFE map of the LMC, similar to Fig. 6, using OGLE III data.

the technique is about $120 \mathrm{Myr}$ and we did not consider regions that have an older turn-off. These regions are expected to appear as gaps in the map. It can be seen that these gaps do not appear in the inner regions, but do appear towards the periphery. In the case of map A, the outer regions are missing for the above reason. If we inspect maps $\mathrm{B}$ and $\mathrm{C}$, we can see that more and more outer regions are covered in these maps. This is because the outer regions have older turn-offs and their ages become younger when the area becomes larger. The point to be noted is that most of the regions in the inner $3^{\circ}$ have experienced star-formation in the last 100 Myr. Another result derived from these maps is that the age of the LSFE gets older with radius. The average age is around $20 \mathrm{Myr}$ in the central regions, whereas it is about $80 \mathrm{Myr}$ near the periphery. These values are found to be more or less similar for all the three area bins.

Figure 7 shows the age map of the LSFE as estimated from the OGLE III data. Map A has the highest spatial resolution and shows a clumpy distribution of ages. The central region, which includes the bar region and the 30 Doradus region, are found to have young ages. These sites have continued to form stars until quite recently. As we found in the MCPS map, we see that the star forming regions have shrunk to increasingly smaller pockets with time, where the star-formation still continues. These pockets corresponds to the bar region and the 30 Doradus region. The spiral-type pattern one could see in the western side of the bar in Fig. 6, is found to break into smaller multiple regions with star-formation. Since the OGLE III has lesser coverage of the north, we are unable to study the northern star forming regions. We see a gradual increase in the age of the LSFE towards the outer regions. The maps $\mathrm{B}$ and $\mathrm{C}$ are obtained with larger area bins and we can see that the details disappear in these plots. On the whole, we find that the central regions have an average age of the LSFE of $20 \mathrm{Myr}$, whereas in the periphery the average age is about 80 Myr. This is similar to what was found in the MCPS maps. Since the OGLE III maps have the highest spatial resolution, we tried to identify any propagating star-formation in the bar region. We do not detect any propagation along the bar. All along the bar, from the northwest end to the southeast end, we detect pockets of very young stars, as young as $\leq 10 \mathrm{Myr}$. These pockets are surrounded by slightly older stars ( $20 \mathrm{Myr})$, as seen in map A. The medium resolution map, B also shows that young stars are distributed right across the bar suggesting the star-formation has been active all along the bar in small pockets. Map A has missing regions towards the periphery which means that in these regions the age of the LSFE is older than $120 \mathrm{Myr}$. As for Fig. 6, maps $\mathrm{B}$ and $\mathrm{C}$ cover more of the outer regions, owing to their larger bin areas.

The maps obtained from both the data sets suggest that the inner regions have continued to form stars up to $\leq 10 \mathrm{Myr}$, whereas the outer regions stopped forming stars earlier ( $~ 80 \mathrm{Myr})$. This suggests that there has been an inward quenching of star-formation. The outer regions are older with an age of about $80 \mathrm{Myr}$, implying that the star-formation stopped at around this age. The younger ages of the LSFE for the inner regions suggest that the star formation has stopped relatively recently or is still continuing. The younger LSFE regions located in the inner regions have a clumpy distribution which suggests that the star-formation has broken up into smaller pockets. Around these pockets, one can see a gradation in the age with relatively older stars being located in the periphery. To summarise, the age maps suggest an outside-to-inside quenching of star-formation in the inner $3^{\circ}$ of the LMC, in the age range 80-1 Myr, with the inner regions experiencing star formation until very recently. Even though three maps correspond to three different area bins and hence the estimated age of the LSFE differ slightly, the above result is seen in all the maps, with varying details.

The statistical distribution of the age of the LSFE in the LMC is shown in Fig. 8. The histograms in three colours represent 
G. Indu and A. Subramaniam: The star-formation history of the Magellanic Clouds

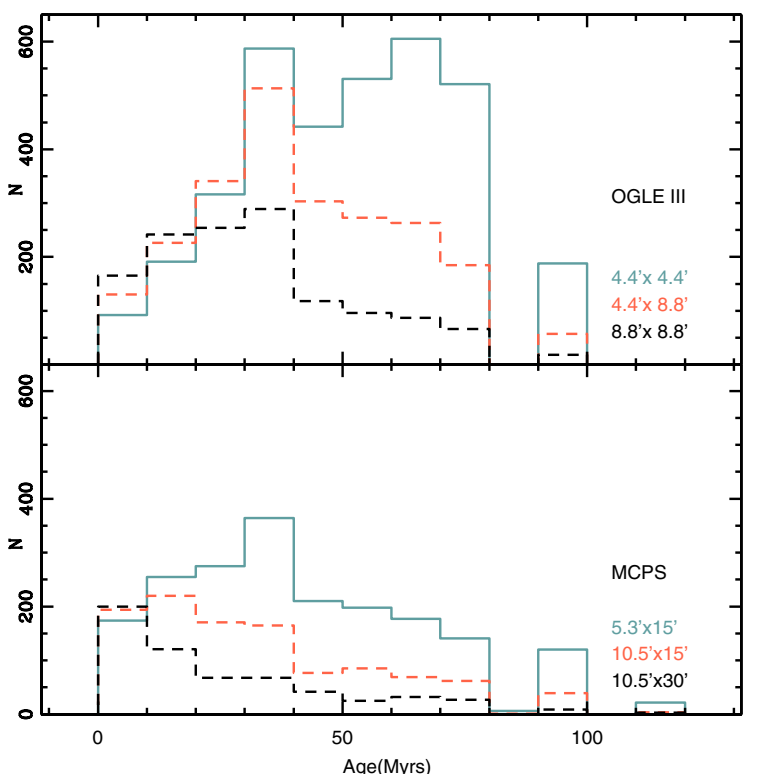

Fig. 8. The statistical distribution of LSFE ages of the LMC. Upper panel shows OGLE III data and lower panel shows MCPS data, with the three colors corresponding to different area, as specified in the figure.

the distribution of ages derived with three different area bins as shown in the figure. In both the distributions obtained using OGLE III and MCPS (upper and lower panels in the figure), it is seen that as we go to higher and higher spatial resolution, the peaks of the distribution tends to shift towards older ages. This can be explained in terms of the number of stars present in a subregion of a particular area. As we create finer bins, the number of stars in the MS of the CMD becomes increasingly smaller, producing in turn an older MS turn-off. In the case of OGLE III, the age of the LSFE peaks at 60-70 Myr and 30-40 Myr, for small area bins. In the case of large area bins, the peak is found only at 30-40 Myr. The time at which the largest number of regions stopped forming stars (30-40 Myr) is similar for all the three area bins. Since OGLE III scans do not cover the northern star forming regions, the above distribution is applicable to the central regions, including the bar. Thus, we might conclude that most of the central regions stopped forming stars at about 30-40 Myr. All the area bins also show an isolated peak at 90100 Myr. This peak is also found in the MCPS distribution for all the area bins. This peak may be similar to the 100 Myr starformation peak identified by H\&Z09.

For the MCPS data, we see that the smallest area bin shows a peak at 30-40 Myr, whereas the largest area bin shows a peak at $0-10$ Myr. We can clearly see the progressive shifting of the peak to younger ages with the increase in the area binned. The medium resolution map shows that the peak is in the range 0-40 Myr. Thus, our analysis of MCPS data which have a larger area coverage finds that the star formation stopped at 0-10 Myr for most of the regions. Thus, the influence of the northern regions is to make the peak shift to younger ages, suggesting that the star-formation in the northern regions continued to younger ages, than in the central regions. Thus, we find a peak of star-formation at $0-10 \mathrm{Myr}$, as for the $12 \mathrm{Myr}$ star-formation peak found by H\&Z09, for the MCPS data. The peaks of starformation identified here coincide with those found by H\&Z09, even though the methods used are different. The above finding also suggests that the ages derived by this method are comparable to those derived by them.

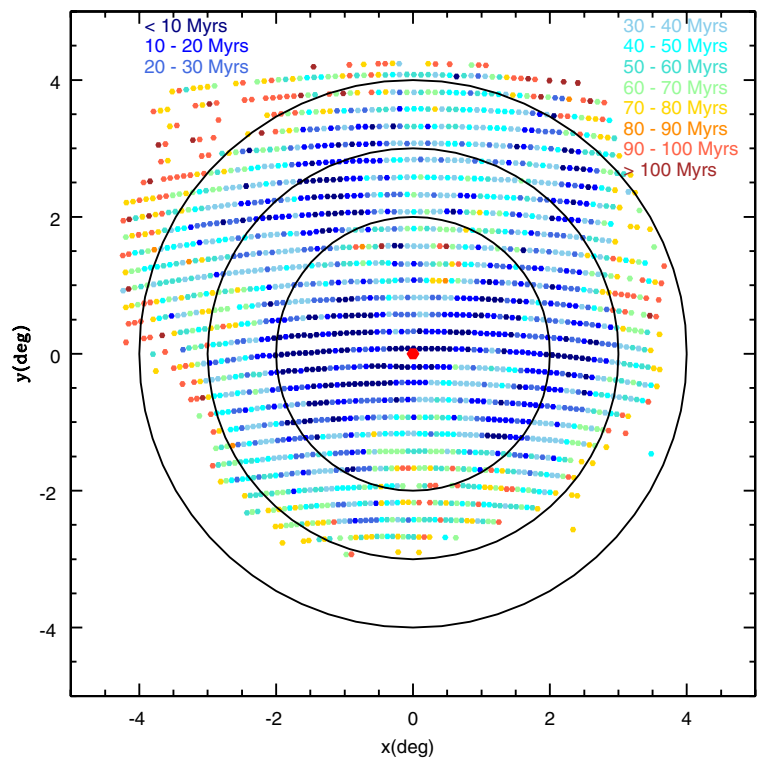

Fig. 9. The LSFE map of the LMC(same as A in Fig. 6) in the projected $x-y$ plane of the sky. Three concentric rings of radii $2^{\circ}, 3^{\circ}$, and $4^{\circ}$ are overplotted.The red dot at $(0,0)$ is the optical center of the LMC.

Among the maps presented above, the OGLE III maps help us to understand the finer details of star-formation in the central regions, because of the higher spatial resolution (with OGLE III we obtain the smallest area for subregions). The MCPS maps cover a larger area, in particular, for the northern regions. It can be seen that there are a number of star forming regions in the north of the LMC, but there are very few in the southern LMC. About the optical center at RA $=5^{\mathrm{h}} 19^{\mathrm{m}} 38^{\mathrm{s}} ;$ Dec $=-69^{\circ} 27^{\prime \prime} 5.2^{\prime}$ (J2000.0 de Vaucouleurs \& Freeman 1973), one can discern a lopsidedness in the recent star-formation towards the northern regions. That is, the quenching of star-formation is asymmetric with respect to the center of the LMC. The quenching appears to have been more effective in the southern LMC, than in the northern part. To substantiate this finding, we plot the LSFE age map (the high resolution map (A) in Fig. 6) in the xy plane using the optical center to convert the RA-Dec to $x-y$ coordinates (Fig. 9). We also show concentric circles with radii of $2^{\circ}, 3^{\circ}$, and $4^{\circ}$ with respect to the center. In the southern regions, it can be seen that older ages are contained within the inner circles, between radii $2^{\circ}$ and $3^{\circ}$. In the northern regions, older star forming regions appear only in the outermost annulus and outside $4^{\circ}$. This map clearly shows the lopsidedness and the extension of the younger star forming regions to the north and the northeast. The map also suggests that the southern regions are more or less symmetric with respect to the center. This result is consistent with the presence of star forming regions such as LMC1, LMC5, LMC4, and the super giant shells in the north, and 30 Doradus in the northeast, whereas similar regions are not found in the southern LMC. The map presented above is in the sky plane and needs to be deprojected onto the LMC plane, to help enhance any lopsidedness. That is, in order to understand these features, one needs to study their location in the plane of the LMC and not in the sky plane. We describe this approach in the following section.

\subsubsection{Deprojection of data onto the LMC plane}

To study the lopsidedness of the age distribution, we need to deproject the data to obtain the distribution in the plane of the 


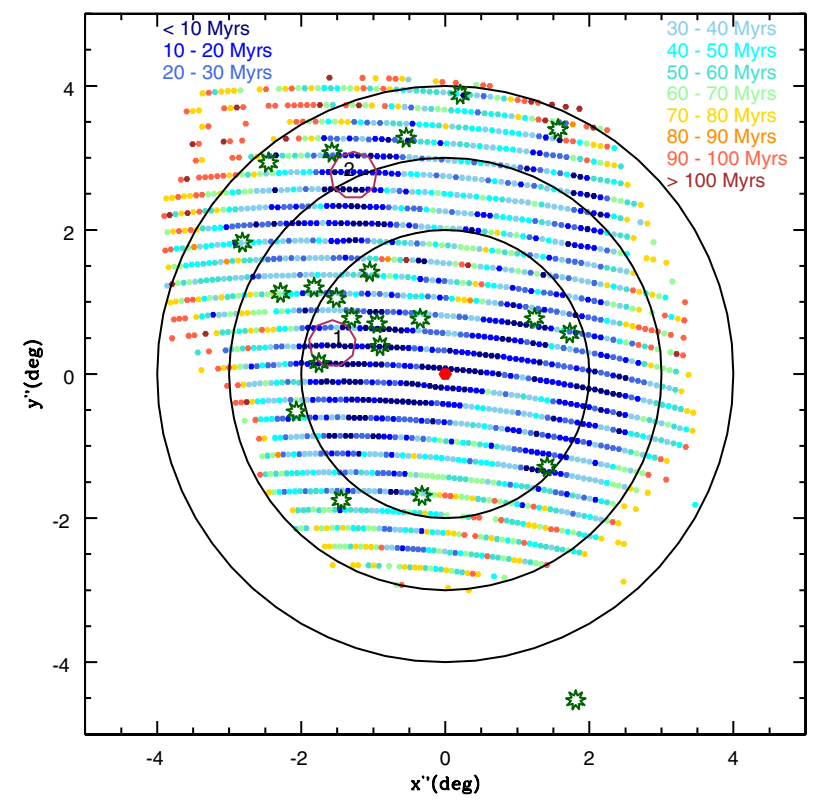

Fig. 10. The LSFE map of the LMC which is similar to Fig. 9, in the deprojected $x^{\prime \prime}-y^{\prime \prime}$ plane of the LMC, where concentric circles are drawn centered on the optical center with radii as in fig 9. The relevant features identified in the LMC plane are shown as hexagons. The numbering is decoded as 1. 30 Doradus, 2. Constellation III (Meaburn 1980), The dark green points are the HI super giant shells (Kim et al. 1999).

LMC. The LMC plane is inclined with respect to the sky plane by an angle $i$ (the face-on view corresponds to $i=0$ ) and the PA of the line of nodes (measured counter-clockwise from the north) is $\Theta$. The near side of the LMC plane lies at $\Theta_{\text {near }}=\Theta-90$ and the far side $\Theta_{\text {far }}=\Theta+90$. Conversion of RA-Dec $(\alpha, \delta)$ to Cartesian coordinates $x-y$ (van der Marel \& Cioni 2001) is done using the conversion equation

$x(\alpha, \delta)=\rho \cos (\phi)$,

$y(\alpha, \delta)=\rho \sin (\phi)$,

where $\rho$ and $\phi$ are the angular coordinates of a point defined by the coordinates $(\alpha, \delta)$ in the celestial sphere, where $\rho$ is the angular distance between the points $(\alpha, \delta)$ and $\left(\alpha_{0}, \delta_{0}\right)$ which is defined to be the center of the LMC, and $\phi$ is the position angle of the point $(\alpha, \delta)$ with respect to $\left(\alpha_{0}, \delta_{0}\right)$. By convention, $\phi$ is measured counter-clockwise starting from the axis that runs in the direction of decreasing RA, at constant declination $\delta_{0}$. Correction for the PA and $i$ can be applied if we know the mean distance to the LMC center $D_{0}$, using the conversion equations

$$
\begin{aligned}
x^{\prime} & =\frac{D_{0} \cos \left(\phi-\Theta_{\mathrm{far}}\right) \sin \rho \cos i}{\cos i \cos \rho-\sin i \sin \rho \sin \left(\phi-\Theta_{\mathrm{far}}\right)}, \\
y^{\prime} & =\frac{D_{0} \sin \left(\phi-\Theta_{\mathrm{far}}\right) \sin \rho}{\cos i \cos \rho-\sin i \sin \rho \sin \left(\phi-\Theta_{\mathrm{far}}\right)} .
\end{aligned}
$$

It is useful in practice however not to use the coordinates in the LMC disk plane, but a new system, in which the line of nodes lie at the same angle in the $\left(x^{\prime \prime}, y^{\prime \prime}\right)$ plane of the LMC, as in the projected $(x, y)$ plane of the sky. It is obtained by rotating $\left(x^{\prime}, y^{\prime}\right)$ by an angle $\Theta_{\text {far }}$

$x^{\prime \prime}=x^{\prime} \cos \Theta_{\mathrm{far}}-y^{\prime} \sin \Theta_{\mathrm{far}}$,

$y^{\prime \prime}=x^{\prime} \sin \Theta_{\mathrm{far}}+y^{\prime} \cos \Theta_{\mathrm{far}}$.

The deprojected MCPS map is shown in Fig. 10, where concentric circles of radii $2^{\circ}, 3^{\circ}$ and $4^{\circ}$ are also shown to compare the

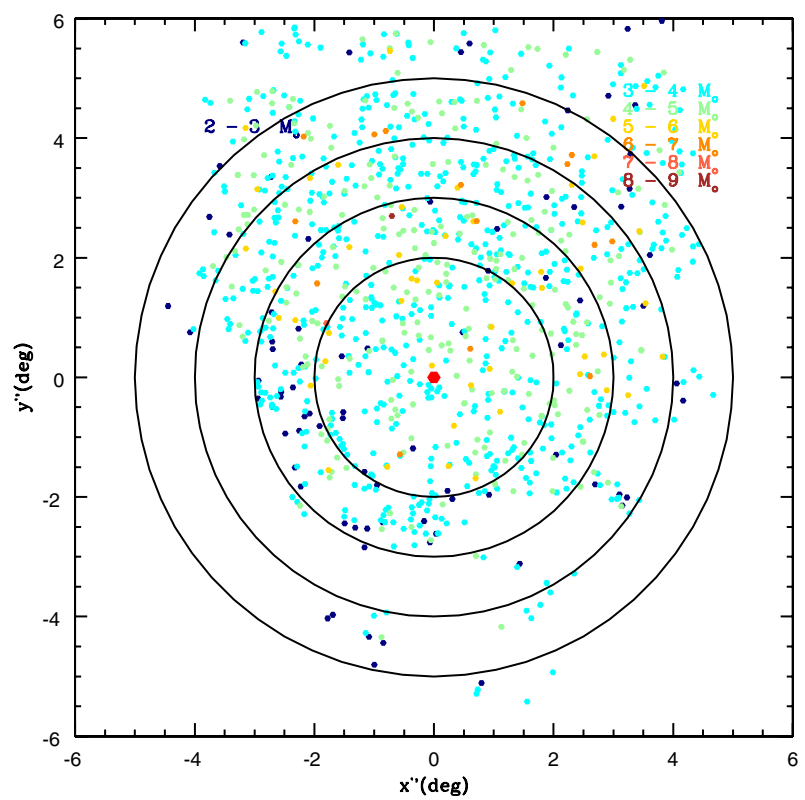

Fig. 11. Map of HI clouds in the LMC plotted in $x^{\prime \prime}-y^{\prime \prime}$ plane. Colour coding is according to the mass in log scale, as specified in the figure. Data is from Kim et al. (2007). The LMC optical center is shown as a red point. Concentric rings are overplotted at radii $2^{\circ}, 3^{\circ}, 4^{\circ}$, and $5^{\circ}$.

distribution with Fig. 9. This map also shows the lopsidedness, suggesting an extension in the north and northeastern directions. If we consider regions in which star-formation stopped around $40 \mathrm{Myr}$ or younger, the location of such regions gives an impression that the star formation is being stretched in the northeastsouthwest direction, with more such regions located in the northeast, with respect to the LMC center. Thus, in the plane of the LMC, the recent star-formation has a lopsidedness towards the north and northeast, which was also detected less clearly in earlier maps. The direction in which the distribution appears to be stretched is in the direction of our Galaxy. van der Marel \& Cioni (2001) found an elongation in the outer stellar distribution of the LMC disk, when viewed in the LMC plane. This elongation of the recent star forming regions is also in the similar direction. We compared this lopsidedness with the distribution of HI gas in the following section.

\subsubsection{Comparison with $\mathrm{HI}$ clouds and star clusters}

The results obtained above suggest that the recent star-formation in the LMC has a lopsidedness in the north and northeast direction. One would also then expect to see a similar lopsidedness in the distribution of the HI clouds in the LMC plane. We have plotted the HI clouds using the data from Kim et al. (2007) in the LMC plane in Fig. 11. The colour code used is according to the mass of the cloud, as indicated in the figure. Concentric circles at $2^{\circ}, 3^{\circ}, 4^{\circ}$, and $5^{\circ}$ are also shown. The deprojected HI distribution is seen to be lopsided with respect to the center. Most of the clouds are located to the north of the center, with very few clouds in the south. The clouds are located within a radius of $3^{\circ}$ in the south, while their distributions extend outside a radius of $5^{\circ}$ in the north. The massive HI clouds are also preferentially populated in the north compared to the south. Thus, the distribution of the HI clouds show lopsidedness towards the north. The age maps and the HI distribution correlate well with each other and point to an extension of the LMC disk towards the north with respect to the optical center. On the other hand, the recent 


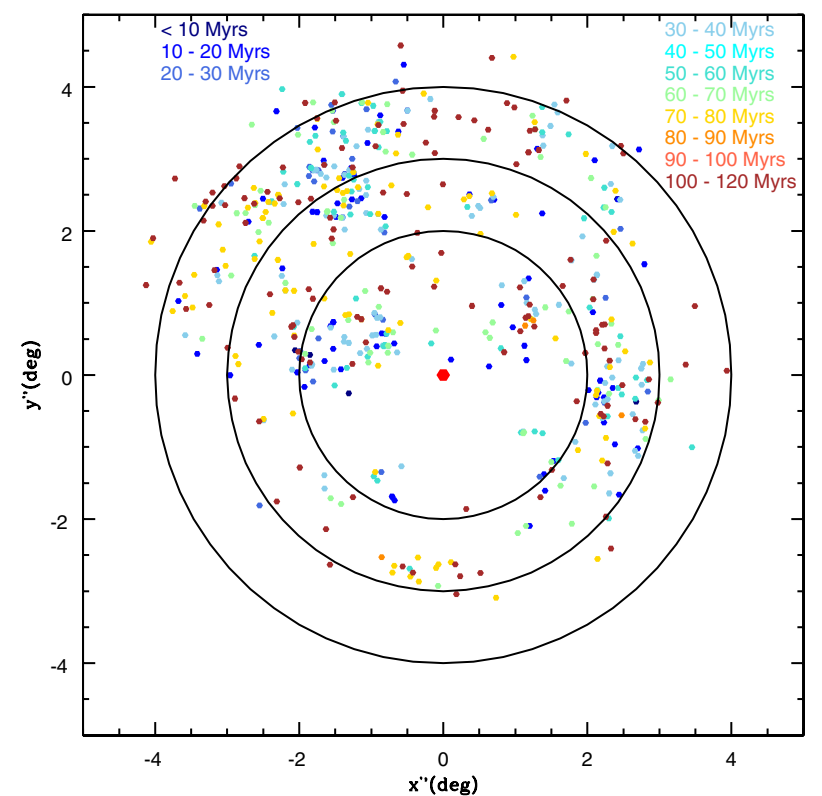

Fig. 12. The spatial distribution of young clusters $(<120 \mathrm{Myr})$ from Glatt et al. (2010) is plotted in the deprojected plane of the LMC. Color coding is according to cluster age as specified in the figure. The red dot is the optical center of the LMC. Concentric rings are drawn at radii $2^{\circ}$, $3^{\circ}$, and $4^{\circ}$.

star-formation also shows a northeast extension. This might suggest that the star-formation is more efficient in the north as well as the northeast in converting gas to stars.

The spatial distribution of young clusters (age $<120 \mathrm{Myr}$ ) in the LMC plane is shown in Fig. 12. The optical center is shown along with concentric rings at radii $2^{\circ}, 3^{\circ}$, and $4^{\circ}$. The cluster distribution is also found to be lopsided. The clusters in the age range $60-100 \mathrm{Myr}$ are distributed out to $3^{\circ}$ in the south, whereas they can be seen out to $4^{\circ}$ and slightly beyond in the north. The clusters in the above age range show lopsidedness towards north. The distribution of clusters younger than $40 \mathrm{Myr}$ is found to shrink within inner regions and are concentrated at the ends of the bar and in the northern regions. We can also see a large concentration of clusters in the northeast. Thus the young ( $\leq 40 \mathrm{Myr}$ ) clusters seem to accumulate in the northeastnorth direction, except for a small group in the southwest of the bar, probably owing to the presence of the bar. We conclude that clusters in the age range 60-100 Myr show lopsidedness towards the north, whereas clusters younger than 40 Myr show lopsidedness towards north and northeast. This might suggest that the northeast enhancement in the star-formation is likely to have happened in the last $40 \mathrm{Myr}$, whereas the northern enhancement has been visible for the last $100 \mathrm{Myr}$.

To compare the ages of LSFE estimated here with the ages of clusters, we have plotted clusters in three age groups in Fig. 13. The LSFE age map only shows the age of the last starformation event and does not suggest anything about previous star-formation episodes in the region. Therefore, while comparing with the cluster ages, we expect the ages of the youngest clusters in a given region to closely match the LSFE ages. In the figure, clusters younger than $40 \mathrm{Myr}$ are shown in the top-left panel. We see a good correlation between the locations of clusters and subregions in this age range such that clusters are located near subregions with similar ages. This also suggests that the ages estimated for the subregions are similar to the ages of the youngest clusters in the vicinity. We also note that there are
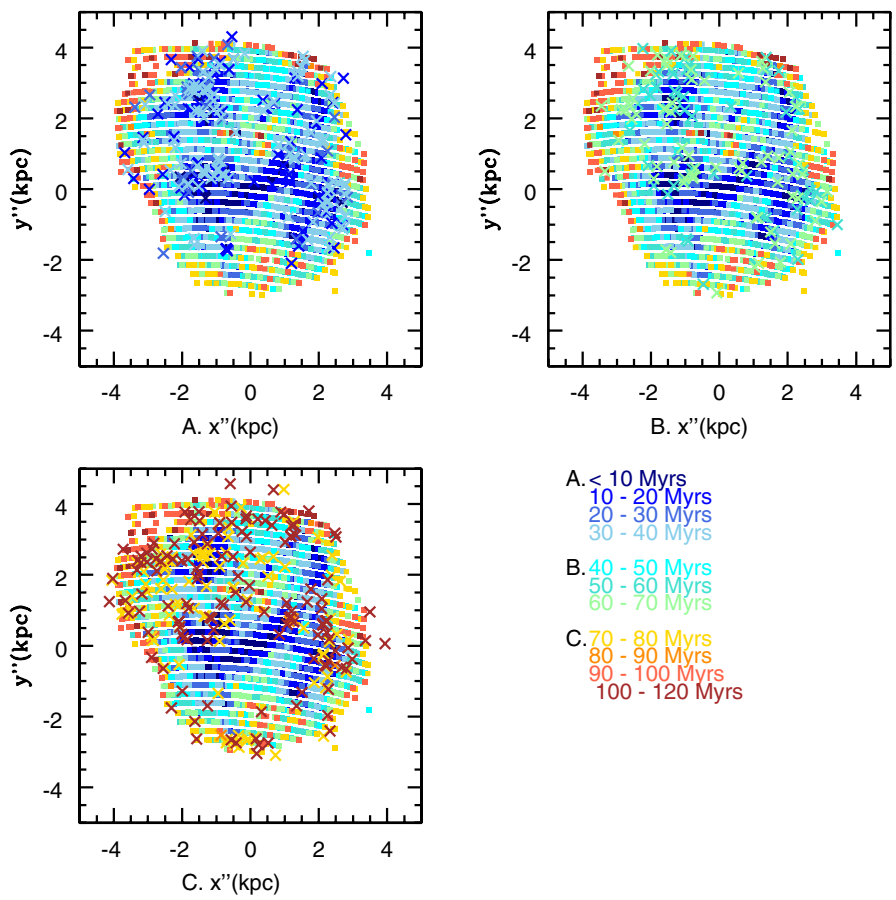

Fig. 13. The age distribution of young clusters (from Glatt et al. 2010) are overplotted on the LSFE map of the LMC in the deprojected $x^{\prime \prime}-y^{\prime \prime}$. Three different age groups are shown, top left, $\leq 40 \mathrm{Myr}$, top right, 40 $70 \mathrm{Myr}$ and bottom left, 70-120 Myr. Color coding is according to the age as specified in the figure.

some regions in the bar that have been forming stars until quite recently, but that there is no cluster formation in these regions. The plot on the top-right panel shows clusters in the range 40$70 \mathrm{Myr}$, and these clusters are distributed as for the younger clusters. We do not see any correlation as most of these clusters are located in regions that have continued to form stars and younger star clusters. In a few regions in the northwest, east, and south, we see similar ages for cluster and the nearby subregions. The bottom-left panel shows the distribution of clusters in the age range 70-120 Myr. These clusters are distributed within a comparatively larger radius, than the young clusters. The ages of these clusters closely match the ages of the subregions in the outer regions of the map. Thus, we find that the age distribution of the LSFE closely agrees with the age distribution of the clusters. The cluster distribution also suggests the star forming regions have shrunk to smaller pockets in the inner LMC, in the last 100 Myr.

\subsection{Shift in the center of the young stellar distribution}

The LSFE age maps suggest that the young star forming regions are found to be lopsided to the north and northeast. The distribution of young clusters as shown in Fig. 12 suggested that the clusters younger than about 40 Myr show a preferential location to the north and northeast. The HI distribution suggests that most of the gas is located in the northern LMC disk. Thus, the center of recent star-formation seems to have shifted to either the north or the northeast, with respect to the optical center. To study this shift in the distribution of the young population as a function of age, we used the MCPS data. Since the number of clusters in a given age range is small, the estimation of the positions of the centroids was not done with clusters. 
Table 1. The centers of the stellar population in the LMC for various ages, using MCPS data.

\begin{tabular}{lccccccc}
\hline \hline Age (Myr) & RA(deg) & Dec $(\mathrm{deg})$ & $x^{\prime \prime}(\mathrm{kpc})$ & $\sigma x^{\prime \prime}$ & $y^{\prime \prime}(\mathrm{kpc})$ & $\sigma y^{\prime \prime}$ & $\mathrm{N \#}$ \\
\hline 8 & 80.5303 & -68.5728 & -0.2598 & -0.0137 & 0.8000 & 0.0160 & 13327 \\
18 & 80.4294 & -68.6190 & -0.2218 & -0.0080 & 0.7584 & 0.0094 & 36536 \\
40 & 80.3217 & -68.7290 & -0.1756 & -0.0046 & 0.6505 & 0.0052 & 107243 \\
60 & 80.3163 & -68.8027 & -0.1666 & -0.0034 & 0.5780 & 0.0038 & 192619 \\
93 & 80.3297 & -68.8768 & -0.1632 & -0.0025 & 0.5051 & 0.0027 & 353207 \\
214 & 80.3542 & -68.9608 & -0.1589 & -0.0014 & 0.4184 & 0.0015 & 1141153 \\
493 & 80.3146 & -68.9201 & -0.1451 & -0.0009 & 0.4470 & 0.0010 & 3151134 \\
\hline
\end{tabular}

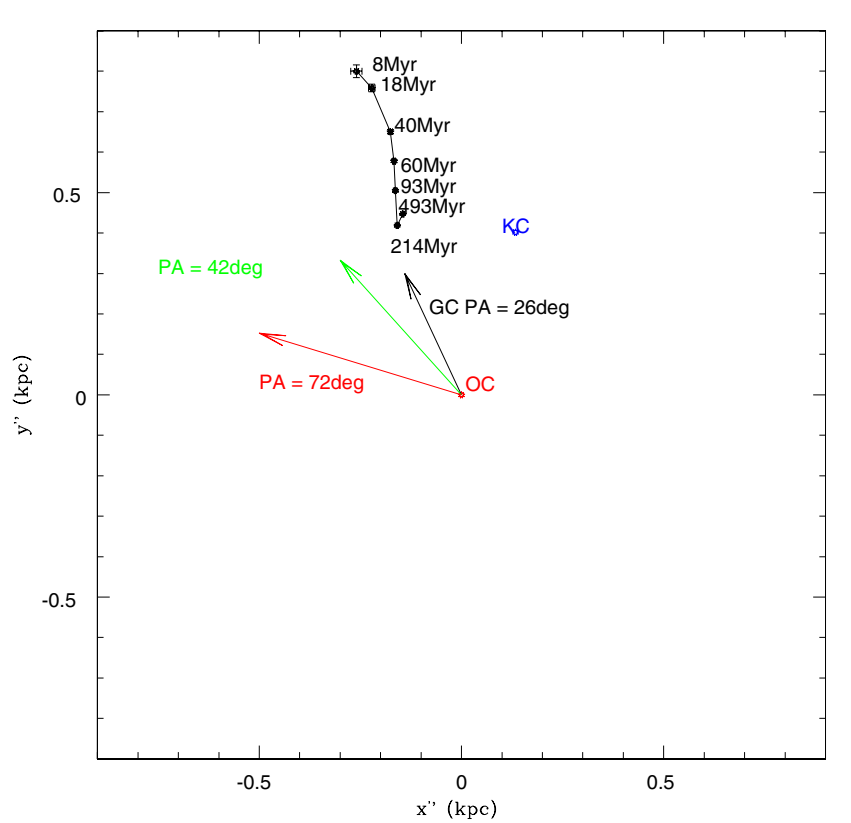

Fig. 14. The centers of the stellar population in the LMC for various ages, is shown in the plane of the LMC, with error bars. The direction of the velocity vector of the LMC is shown in red at a position angle of $72^{\circ}$, (calculated from the proper motion values provided by Piatek et al. 2008) and the line of interaction of the MW and the LMC according to our convention at a position angle of $26^{\circ}$. The direction given in van der Marel (2001) is shown in green. $\mathrm{KC}$ is the HI kinematic center (Kim et al. 1998) shown in blue, and OC is the optical center of the LMC shown as a red point.

We used MS star counts to estimate the distribution of stars younger than a particular age using the MCPS data. In the MS, stars brighter than a cut-off magnitude are identified. These stars are younger than the age corresponding to the cut-off magnitude. The center of the distribution of these stars are estimated in the sky plane (RA vs. Dec plane), as well as the LMC plane ( $x^{\prime \prime}$ vs. $y^{\prime \prime}$ plane). The age tagged with such a population is the age of the oldest population in the group, even though there are stars younger than this age in that group. Table 1 contains the age of the oldest population of the group, centers in RA and Dec, and $x^{\prime \prime}$ and $y^{\prime \prime}$, the number of stars considered for the center estimation and the error in the values of the center. Both $x^{\prime \prime}$ and $y^{\prime \prime}$ are in $\mathrm{kpc}$ where $1^{\circ}$ is equal to $0.89 \mathrm{kpc}$ at the distance of the LMC. The centers of stellar population in the LMC plane, for various ages, are shown in Fig. 14. The optical center (OC) and the kinematic center of the gas (KC, taken from Kim et al. 1998) are also shown. The oldest population considered has an age of $500 \mathrm{Myr}$ and the youngest is about $10 \mathrm{Myr}$. The center of the distribution does not shift in the interval 500-200 Myr, even though a small shift towards the south can be noticed. The shift in 200$40 \mathrm{Myr}$ is clearly visible along the $y^{\prime \prime}$ axis, towards the north.
We detect a shift of $7 \mathrm{pc} / 10$ Myr towards the north during the interval 200-100 Myr, while an enhanced shift of $27 \mathrm{pc} / 10 \mathrm{Myr}$ towards the north is detected in the 100-40 Myr age range. On the other hand, no significant shift is present along the $x^{\prime \prime}$ axis (eastwest axis) in the above age range. For populations younger than $40 \mathrm{Myr}$, a shift along both the axes can be noticed suggesting that the center is progressively shifting in the northeast direction. In the 40-10 Myr age range, a shift of $50 \mathrm{pc} / 10 \mathrm{Myr}$ to the north and $28 \mathrm{pc} / 10 \mathrm{Myr}$ to the east are detected. This analysis suggests that the northern lopsidedness in the stellar distribution started between 200-100 Myr. This can be compared to the appearance of the northern blue arm in the age range 160-100 Myr in the SFH by H\&Z09, which could shift the center of the stellar distribution to the north. To summarise, we find that the center of the distribution of stars shifts northward in the age range 200-40 Myr, and that the center is found to shift in the northeast direction for populations younger than $40 \mathrm{Myr}$. This correlates well with the age vs shift found in the cluster distribution. Thus, the stellar population as well as the cluster population has experienced a shift in the northeast direction in the last $40 \mathrm{Myr}$ only. H\&Z09 also finds enhanced star-formation in the northeastern regions for ages $<50 \mathrm{Myr}$, which can be inferred from their Fig. 8.

The line of interaction between the MW and the LMC is shown in Fig. 14. According to our convention, this line is at a position angle of $26^{\circ}$ (shown in black), whereas the direction given in van der Marel (2001) is $42^{\circ}$ (shown in green). This is the direction towards the Galactic center. The direction of the velocity vector of the LMC is shown in red, at a position angle of $72^{\circ}$. It can be seen that the direction of the shift of the center is almost in the direction towards the Galactic center. The LMC disk is known to be inclined such that the northeast part is closer to the Galaxy. The lopsidedness in the star-formation is in the same direction as the inclination. In addition, the LMC is moving past our Galaxy after its closest approach. Thus, the lopsidedness in the stellar as well as the HI distribution to the north may be due to the gravitational attraction of our Galaxy on the gas of the LMC disk and the enhanced compression in the northern regions. The movement of the LMC could cause compression of the gas in the northeastern side resulting in enhanced star-formation in the northeast. The center shifts and the timescales derived in this section can be used to understand the details of the above two processes on the gas resulting in starformation. We discuss these aspects in detail in our discussion section.

\subsubsection{Comparison with the star-formation history of Harris \& Zaritsky (2009)}

A complete star-formation history of the LMC was derived by H\&Z09 using the multi-band photometry of the MCPS. They provided the star-formation rate (SFR) in $M_{\odot} / \mathrm{Myr}$, for particular 

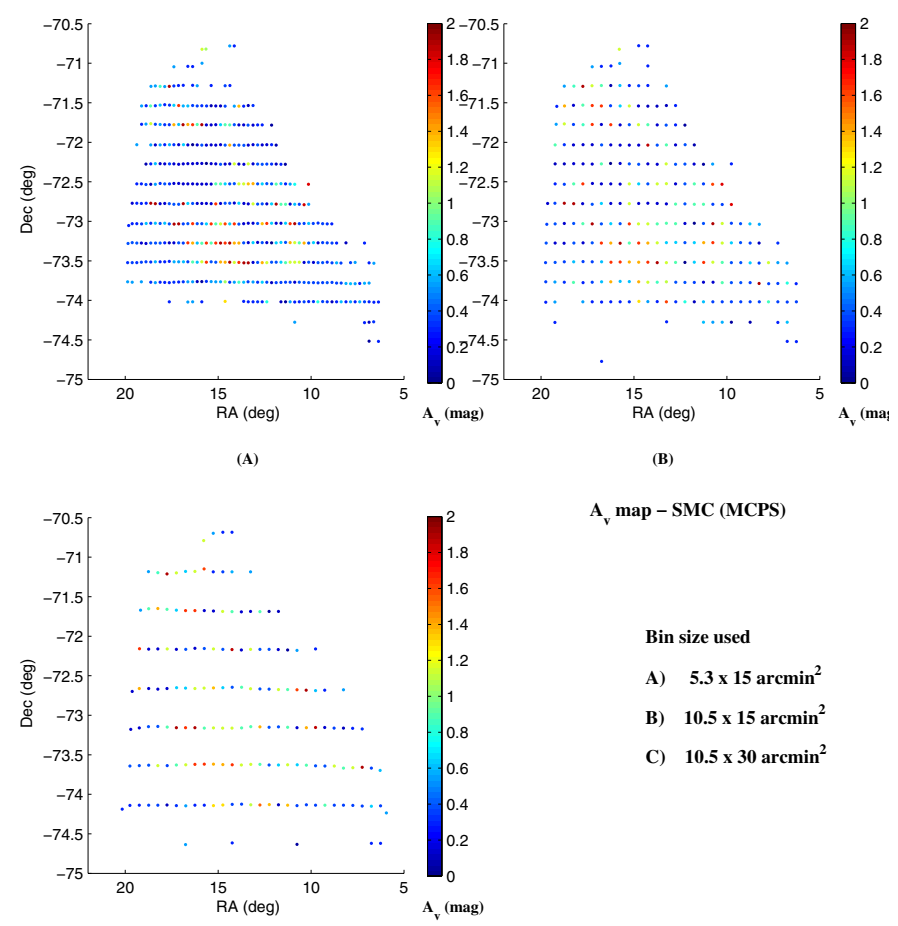

A map - SMC (MCPS)

Bin size used

A) $5.3 \times 15 \operatorname{arcmin}^{2}$

B) $10.5 \times 15 \operatorname{arcmin}^{2}$

C) $10.5 \times 30 \operatorname{arcmin}^{2}$

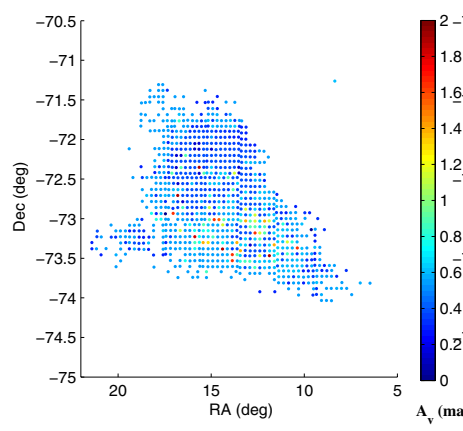

(A)

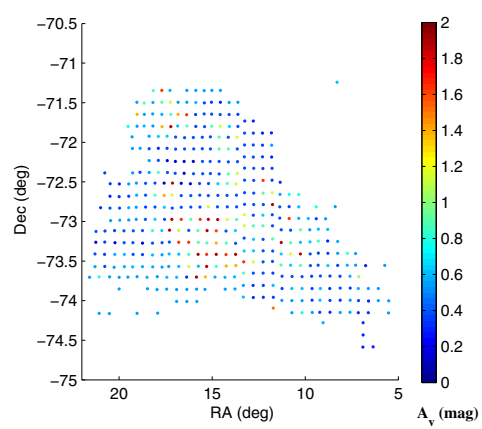

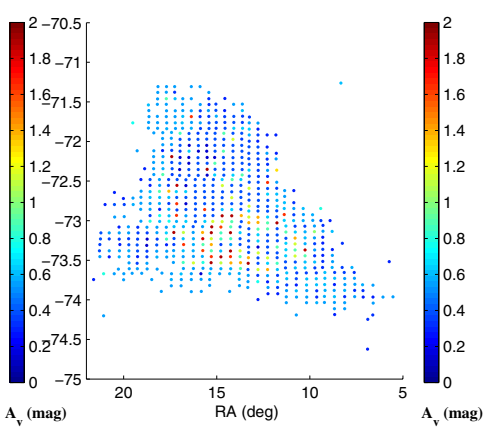

(B)

Fig. 15. The extinction map of the SMC using MCPS data in the RADec plane for three different area binning, as specified in the figure. Color coding is based on the $A_{v}$ value, which varies from 0.2 to 2.0 as shown in the color bar.

age bins for different regions of the LMC. Our analysis identifies the LSFE in a region and estimates its age, which is quite different from the analysis of H\&Z09. Hence, it is impossible to compare the two results quantitatively. On the other hand, we can compare our results with the recent SFH as estimated by H\&Z09. We compare our Fig. 9 with the 12.5 Myr and 6.3 panels of Fig. 8 in H\&Z09. It can be seen that there is a very good correlation between the star forming regions identified in Fig. 8 and those in our Fig. 9. The shrinking of star-formation to smaller regions is clearly seen if we compare their $12.5 \mathrm{Myr}$ and 6.3 Myr panels. They find that the northern blue arm appears in the 160100 Myr age range. We note that there has been a shift in the population to the north in the last $200 \mathrm{Myr}$. In addition, the enhanced star-formation in the northeast regions appear for ages younger than $50 \mathrm{Myr}$ in H\&Z09, whereas we find such an enhancement at 40 Myr. They identified peaks of star-formation at $12.5 \mathrm{Myr}$ and $100 \mathrm{Myr}$, which correlates well with the peaks of 0-10 Myr and 90-100 Myr identified by us. Thus, the ages of the LSFE estimated here correlates well with the recent SFH derived by H\&Z09.

\subsection{SMC: extinction}

The extinction towards the SMC is estimated using the MCPS and OGLE III data sets. As in the case of the LMC, we estimated the reddening $E(V-I)$ of the main sequence stars near the turnoff for each region. The extinction $\left(A_{V}\right)$ maps estimated using the MCPS data are shown in Fig. 15 and those estimated from OGLE III are shown in Fig. 16. The area used for three different bin sizes are mentioned in the figure. The maps are found to be similar with the extinction value in the range, $0.2-2.0 \mathrm{mag}$. The average extinction is found to be in the range $0.2-0.5 \mathrm{mag}$ with central regions showing a higher value of extinction. The eastern

Fig. 16. The SMC extinction map for three area bins, as in Fig. 15, using OGLE III data.

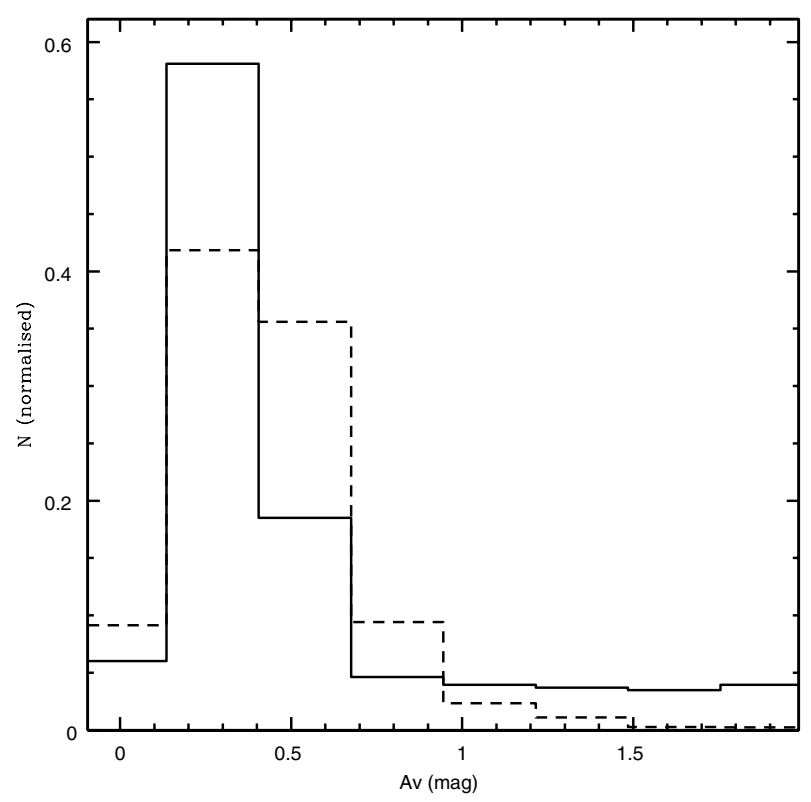

Fig. 17. The estimated distribution of extinction in the SMC (shown as solid line) is compared with distribution obtained from the extinction map of hot stars provided by Zaritsky et al. (2002) (shown as dashed line, extracted from the fits image from the authors' website).

wing also has regions with high extinction. Zaritsky et al. (2002) estimated the extinction across the SMC. We compared our estimation with their values in the form of a distribution, in Fig. 17. In general, both distributions closely match and both have a peak in the range, 0.2-0.4 mag. As for the LMC, we derive lower extinction than Zaritsky et al. (2002), for the reasons mentioned in Sect. 4.1. We do observe a mild inconsistency in the distribution for extinction values higher than $1.5 \mathrm{mag}$. Since the number 


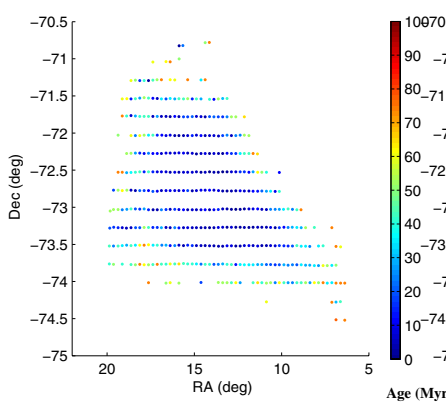

(A)

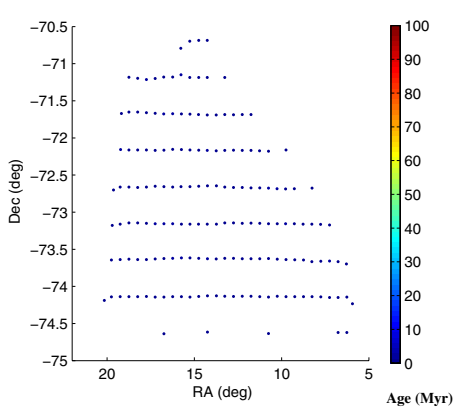

(C)

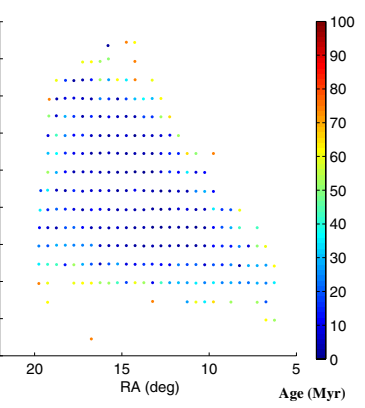

(B)
Bin size used

A) $5.3 \times 15 \operatorname{arcmin}^{2}$

B) $10.5 \times 15 \operatorname{arcmin}^{2}$

C) $10.5 \times 30 \mathrm{arcmin}^{2}$
Fig. 18. The LSFE map of the SMC using MCPS data in the RADec plane with three different area binnings, as specified in the figure. Color coding is based on the LSFE age as shown in the color bar.

of regions involved is small, it is unlikely to affect the results derived below.

\subsection{SMC: age map of the LSFE}

We estimated the spatial distribution of age of the LSFE in RA vs. Dec plane and presented the maps in Figs. 18 (MCPS) and 19 (OGLE III). We adopted three sizes of area (bin sizes as specified in the figure) to estimate the ages and to understand the effect of the area on the estimated age. The MCPS maps show that the central region has experienced star-formation until very recently (0-20 Myr). In the high resolution (smallest area size) map A, the outer regions appear to have older ages of about 60-80 Myr. This is not so clear in the map B, and in map C, we do not see any such increase in the age of the LSFE with radius. This clearly shows the effect of the area used in estimating the age of the LSFE. The OGLE III plots use smaller sizes of area (Fig. 19), and the high resolution map A, shows that the inner region has a substructure with two clumps in the northeast and southwest direction. This substructure is similar in location to the two HI super shells, 304A and 37A (Stanimirovic et al. 1999). The map also suggests that the northeast wing has more regions with recent star-formation, than the southern clump, which is located near the center of the SMC. This structure disappears in the maps B and C. In all the OGLE III maps, we can find that the age of the LSFE in the central regions is in the age range 0-20 Myr, while the periphery shows an age of $~ 60$ Myr. Unlike in the LMC, we do not see a gradient in age, increasing outward from the center, even though the outer regions have older age. We also find that the missing regions caused by the limiting magnitude appear only in the periphery, suggesting that all the inner regions have experienced star-formation in the last 100 Myr. The missing outer regions also suggest that the star-formation stopped in

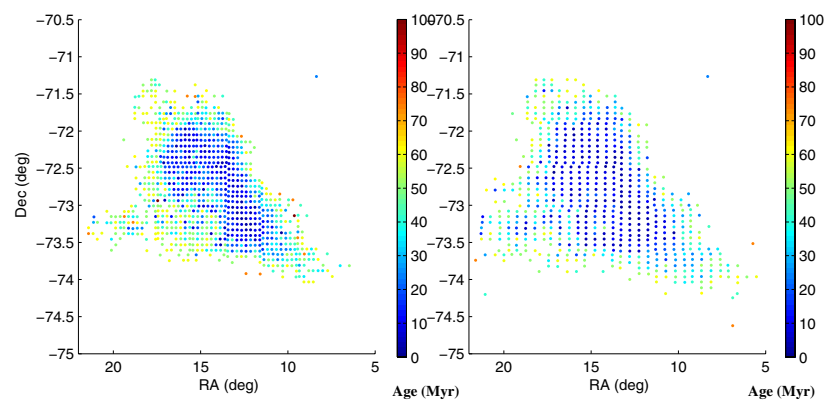

(A)

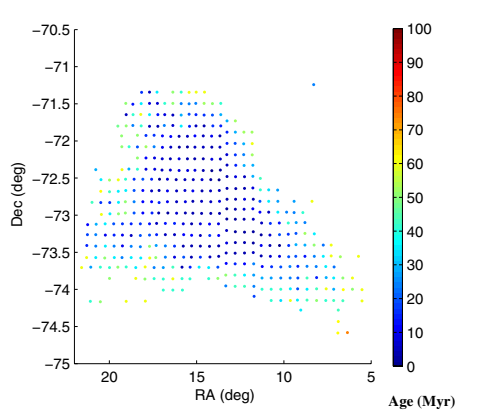

LSFE map - SMC (OGLE III)

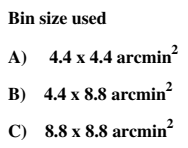

C) $8.8 \times 8.8 \operatorname{arcmin}^{2}$

(C)

Fig. 19. The LSFE map of the SMC similar to Fig. 18, using OGLE III data.

the outer regions much earlier. As the area of the bin (maps B and $\mathrm{C}$ ) increases, more regions get added to the periphery owing to the shift of the LSFE to younger ages. To summarise, we find that the star-formation in the inner SMC is not very structured, in comparison with that in the LMC. We can identify the eastern wing in the map. Most of the inner regions have experienced star-formation in the last 0-20 Myr. As for the LMC, we find marginal evidence of an outside-to-inside quenching of star-formation in the last $60 \mathrm{Myr}$.

The histogram of the age distribution of the LSFE is shown in Fig. 20. All three maps from the OGLE III (upper panel) identify a peak at 50-60 Myr. The younger peak shifts from 10-30 Myr in the highest resolution to 0-10 Myr in the low resolution maps. The shift to younger ages is found as the area considered increases. The distributions obtained from the MCPS map (lower panel) show a mild peak at 50-60 Myr in the two lowest resolutions, whereas the peak widens to 40-60 Myr at high resolution. All three resolutions show a peak at 0-10 Myr. In summary, we find that most of the regions in the SMC have experienced star-formation until very recently, with one peak at 0-10 Myr and another at 50-60 Myr. The SFRs of the SMC are shown in Fig. 19 of H\&Z09, which is derived from the SFH estimated in Harris \& Zaritsky (2004). They identified a peak around $50 \mathrm{Myr}$ and another around $10 \mathrm{Myr}$, which is in good agreement with our finding. Hence, the results derived here correlate well with the recent SFH derived by Harris \& Zaritsky (2004).

\subsubsection{Comparison with the HI gas distribution and star clusters}

We have plotted the HI column density distribution of Stanimirovic et al. (2004) in Fig. 21. The HI has the highest column density in the southwestern part. The LSFE age map (corresponding to the high resolution map A in Fig. 19) is shown 
G. Indu and A. Subramaniam: The star-formation history of the Magellanic Clouds

Table 2. The centers of the stellar populations in the SMC for various ages using OGLE III data.

\begin{tabular}{lccccccc}
\hline \hline Age (Myr) & RA(deg) & Dec $(\mathrm{deg})$ & $x(\mathrm{kpc})$ & $\sigma x$ & $y(\mathrm{kpc})$ & $\sigma y$ & $\mathrm{N \#}$ \\
\hline 6 & 14.2007 & -72.7345 & -0.3631 & -0.0197 & 0.0445 & 0.0128 & 2430 \\
14 & 14.3156 & -72.7533 & -0.3981 & -0.0109 & 0.0472 & 0.0069 & 7968 \\
33 & 14.2957 & -72.7728 & -0.3916 & -0.0063 & 0.0334 & 0.0041 & 23377 \\
79 & 14.1303 & -72.8157 & -0.3413 & -0.0036 & -0.0088 & -0.0024 & 67919 \\
188 & 14.0868 & -72.8496 & -0.3279 & -0.0021 & -0.0425 & -0.0014 & 203383 \\
445 & 13.9135 & -72.9043 & -0.2739 & -0.0011 & -0.0980 & -0.0008 & 597714 \\
\hline
\end{tabular}

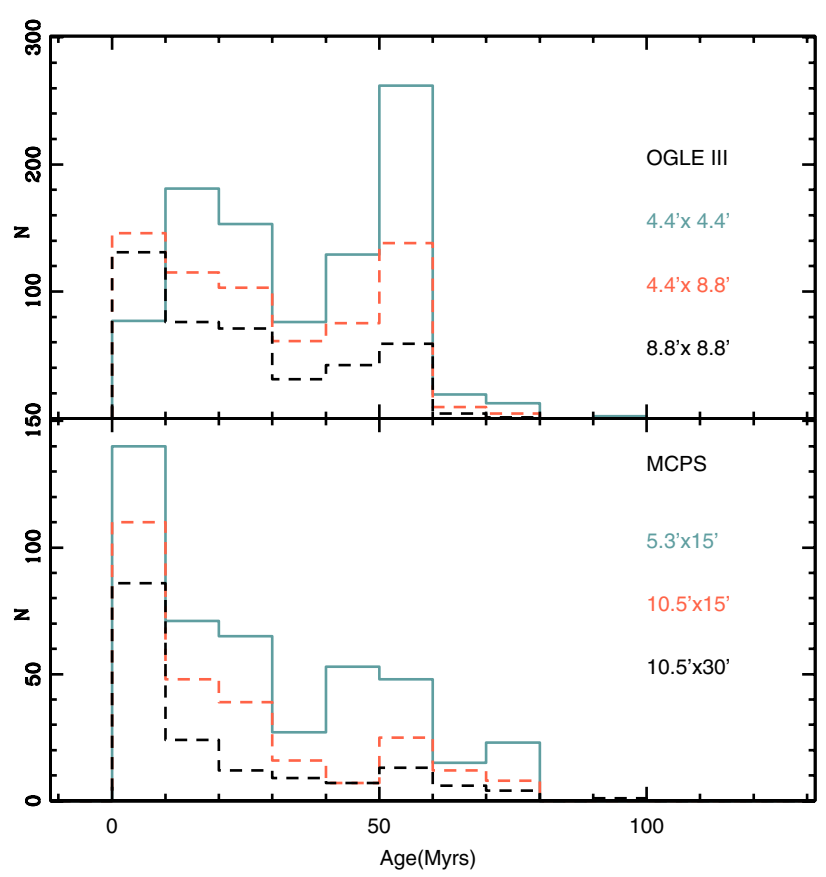

Fig. 20. The statistical distribution of LSFE ages for the SMC. Upper panel shows OGLE III data and lower panel shows MCPS, with the three colors corresponds to different area, as specified in the figure.

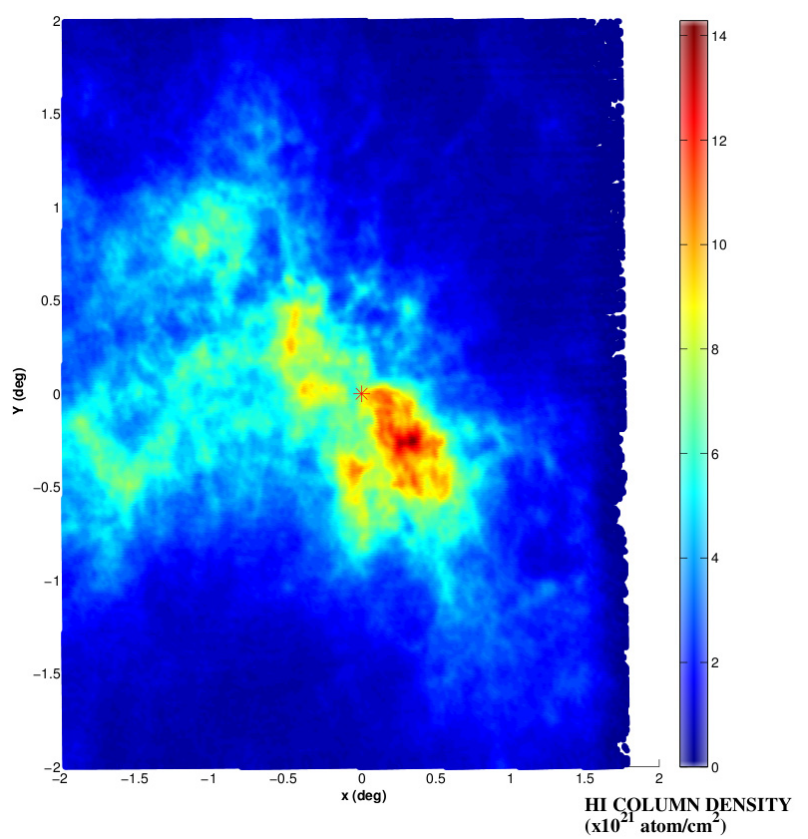

Fig. 21. The HI column density distribution in the SMC is shown in the projected $x-y$ plane of sky. Color coding is according to HI column density as depicted in the color bar. Data is taken from Stanimirovic et al. (2004).

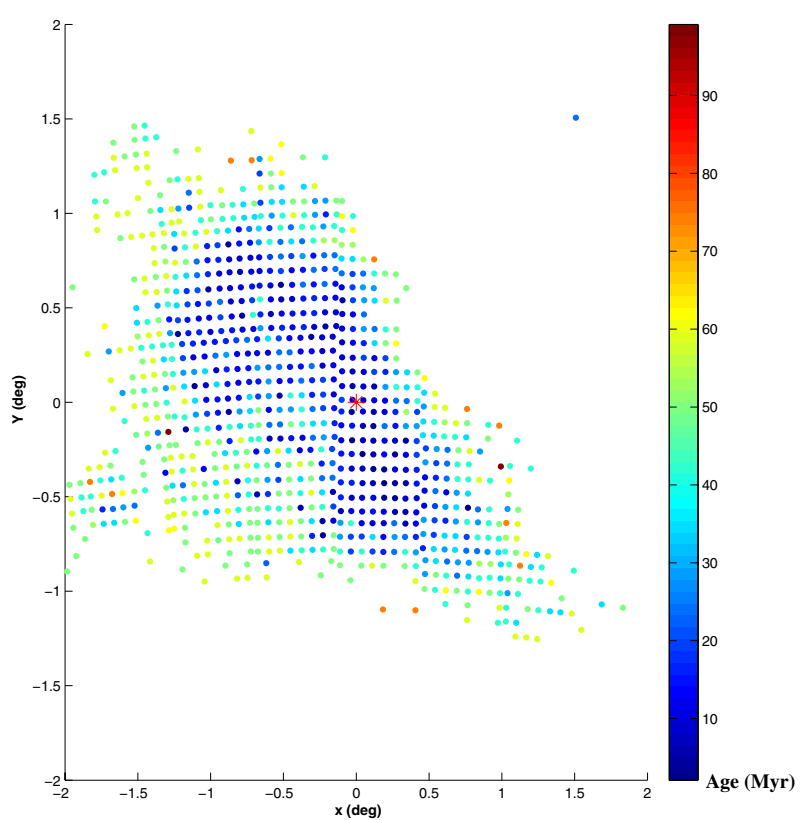

Fig. 22. The LSFE map of the SMC is shown in the projected $x-y$ plane of the sky, as in Fig. 19A. The optical center is shown in red.

in the $x-y$ plane in Fig. 22 for comparison. The northeast and the southwest substructures of the LSFE age map match well with the location of the HI. The LSFE map also identifies young star forming regions in the eastern part of the wing as well as in the northeast part. Overall, the young star forming regions identified here coincide with the locations of HI with column densities higher than $7-8 \times 10^{21}$ atom $/ \mathrm{cm}^{2}$. We identify more regions with recent star-formation in the northeast substructure than the southwest substructure, whereas relatively more/denser $\mathrm{HI}$ is found in the southwest substructure. This suggests that the star-formation is more efficient in converting gas to stars in the northeastern wing. This might also suggest an efficient gas compression in the northeast, similar to the case of the LMC.

The ages of young star clusters given in Glatt et al. (2010) is overplotted on the LSFE maps in Fig. 23. As mentioned in Sect. 4.2.2, while comparing with the cluster ages, we expect that the ages of the youngest clusters in a given region should match with the LSFE ages. The top-left panel shows clusters in the age range 0-40 Myr. In general, these clusters are found to be located near regions of recent star-formation. Clusters in the age range 30-40 Myr located towards the outer regions, are also found near subregions with similar ages. Young clusters $(0-$ $30 \mathrm{Myr}$ ) are found to be located in the northeast and southwest substructures and relatively higher number of clusters are found in the northeast substructure. This suggests that the cluster formation is more efficient in the northeast substructure. The topright panel shows clusters in the age range 40-70 Myr. We note that older clusters near younger star forming regions imply that 

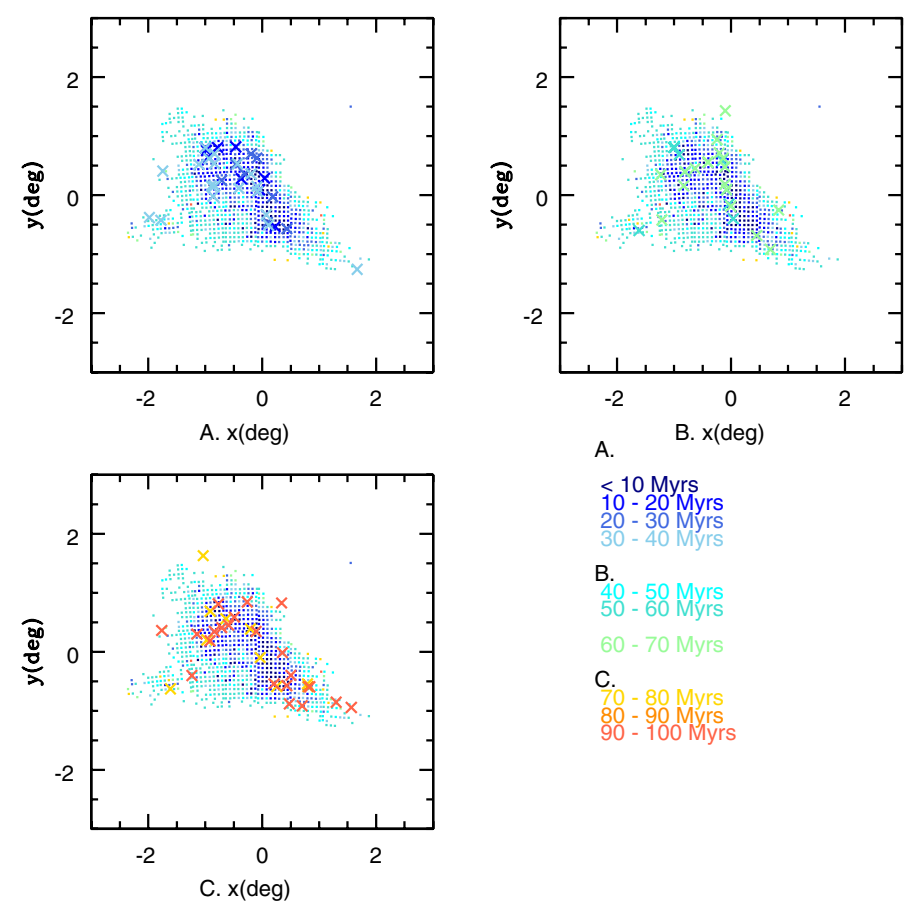

Fig. 23. The age distribution of clusters as old as or younger than 100 Myrs (Glatt et al. 2010) overplotted with the LSFE map (same as Fig. 22) in the projected $x-y$ plane of the sky. Three different age groups are shown, top left $\leq 40 \mathrm{Myr}$, top right $40-70 \mathrm{Myr}$, and bottom left 70-100 Myr. Color coding is according to the age as specified in the figure.

there have been older cluster forming episodes in these regions. Clusters in the age range 70-100 Myr are shown in the bottomleft panel. Since we do not see many regions with LSFE in this age range, no clear comparison is possible. The older clusters are found to occupy a relatively large radial extent compared to the younger clusters. This is indicative of an outside-to-inside quenching of star/cluster formation.

\subsection{Shift in the center of the young stellar distribution in SMC}

We used MS stars younger than various age cut-offs to study the shift of the centroids in the SMC. The data used here is OGLE III, taking into account its higher resolution and larger number of stars. Since the geometry of the SMC is not clearly understood, the center of the distribution of these stars are estimated both in RA-Dec and in the $x-y$ coordinates (with respect to the optical center of the SMC). The optical center is taken as $\mathrm{RA}=0^{\mathrm{h}} 52^{\mathrm{m}} 12.5^{\mathrm{s}} ;$ Dec $=-72^{\circ} 49^{\prime \prime} 43^{\prime}(\mathrm{J} 2000.0$ de Vaucouleurs $\&$ Freeman 1973) The coordinates $x$ and $y$ are in kiloparsec where $1^{\circ}$ corresponds to $1.04 \mathrm{kpc}$ at the distance of the SMC. Table 2 contains the age of the oldest population of the group, centers in RA and Dec, and $x$ and $y$, the number of stars considered for the center estimation, and the error in the values of the center. The oldest population considered is about $450 \mathrm{Myr}$ and the youngest is about $5 \mathrm{Myr}$. The location of the centers, along with the ages and error bars, are shown in Fig. 24. We detect a shift in the center during the time interval 500-200 Myr in the northeast direction. We estimate a shift of $2.1 \mathrm{pc} / 10 \mathrm{Myr}$ along the $x$ and $2.2 \mathrm{pc} / 10 \mathrm{Myr}$ along the $y$ directions. The shift then continues until $30 \mathrm{Myr}$ in the same direction, with an enhanced rate of $4 \mathrm{pc} / 10 \mathrm{Myr}$ along the $x$ direction and $5 \mathrm{pc} / 10 \mathrm{Myr}$ along

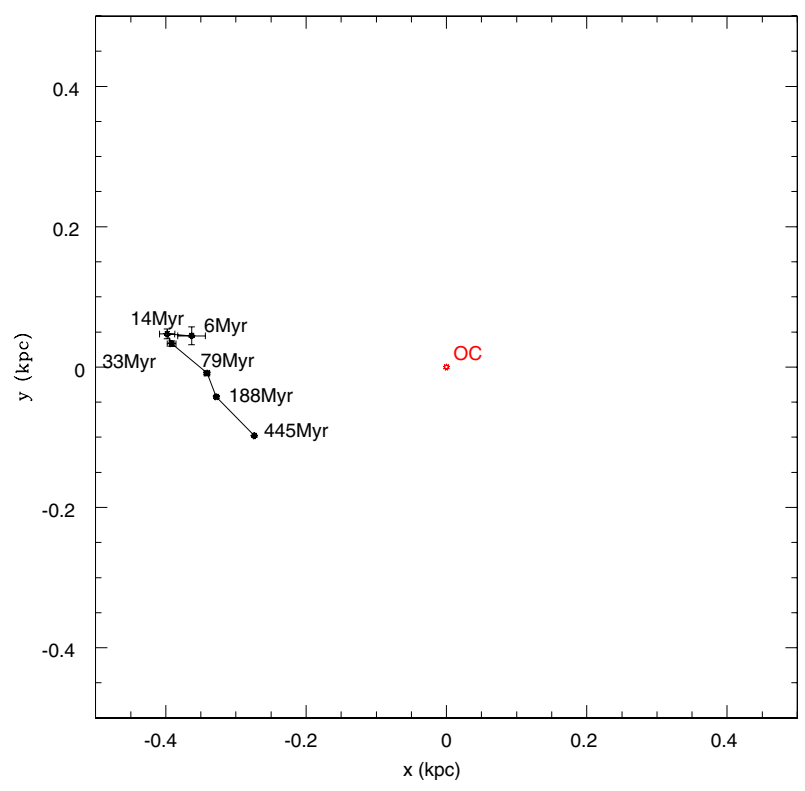

Fig. 24. The figure depicts the locations of the center of the number density distribution of stars tagged with various upper age cut-offs in the projected sky plane for the SMC (Table 2). The error bars are shown and the optical center is shown in red.

the $y$ direction in the 200-30 Myr age range. There is no significant center shift for ages younger than $30 \mathrm{Myr}$. We see a mild shift to the west in the range 14-6 Myr, but to within $2 \sigma$ of the error, as can be seen from Fig. 24. The amount of shift in the center is far smaller than the shift we detected in the LMC. Thus, we detect a significant shift in the center of the population younger than $500 \mathrm{Myr}$ in the northeast direction. This is the direction towards the LMC. H\&Z09 detected a coincident peak of enhanced star-formation at $400 \mathrm{Myr}$ in both the Clouds and they suggested that this may be caused by their mutual interaction. The above center shift during the interval 500-200 Myr may be due to this star-formation episode and enhanced star-formation in the northeastern region, as well as the appearance of the wing in this age range. We detect an enhanced center shift in the 200-30 Myr age range, this could be caused by the gravitational attraction of our Galaxy during the perigalactic passage.

\section{Discussion}

Recent high-precision proper motion estimates suggest that the LMC and the SMC are either on their first passage or on an eccentric long period ( $>6 \mathrm{Gyr}$ ) orbit about the MW. This differs markedly from the canonical picture in which the Clouds travel on a quasi-periodic orbit about the MW (with a period of 2 Gyr). Without a short-period orbit about the MW, the origin of the Magellanic Stream, a young (1-2 Gyr old) coherent stream of $\mathrm{H}$ I gas that trails the Clouds and spans $150^{\circ}$ across the sky, can no longer be attributed to stripping by MW tides and/or ram pressure stripping by MW halo gas (taken from Besla et al. 2010). In addition, the episodes of star-formation found in the LMC have been traditionally attributed to its repeated interaction with the MW and the SMC. Since the SMC is less massive relative to the LMC, it is unlikely to have a significant impact on the LMC. In the first passage scenario, the LMC has probably had its closest approach about $200 \mathrm{Myr}$ ago. This event is likely to have had a significant impact on both the Clouds, especially on the LMC. 
G. Indu and A. Subramaniam: The star-formation history of the Magellanic Clouds

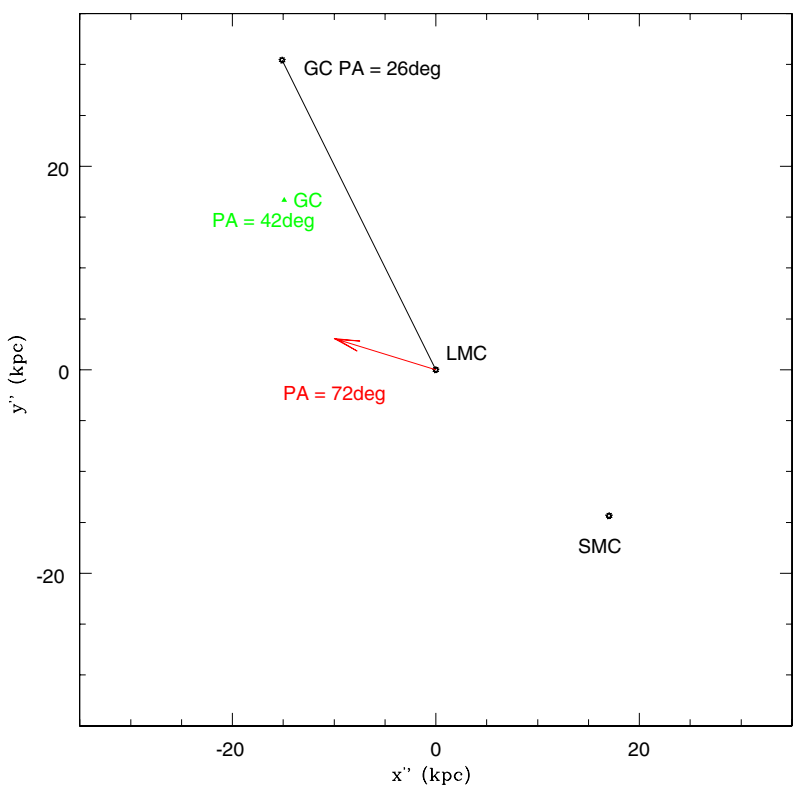

Fig. 25. The locations of the MW, LMC, and the SMC are shown in the plane of the LMC, the direction of the velocity vector of the LMC is shown in red at a position angle $72^{\circ}$, and the line of interaction of the MW and the LMC according to our convention is drawn at a position angle of $26^{\circ}$. The Galactic center taken from van der Marel (2001) is shown in green at a PA $42^{\circ}$. The location of the SMC is such that the LMC lies in the line of interaction of the SMC and the MW.

In the plane of the LMC, we find that the present distribution of the HI gas is lopsided towards the north. The star-formation in the last $40 \mathrm{Myr}$ is lopsided towards both the north and northeast. The center of the stellar population is found to have shifted to the north in the last 200-40 Myr, and towards the northeast in the last 40 Myr. Similarly, H\&Z09 found the appearance of the blue arm around 160-100 Myr ago and the northeastern enhancement for ages <50 Myr. To understand the MW-LMC-SMC interaction, their locations are shown in the plane of the LMC, in Fig. 25. The direction of velocity vector of the LMC is at a position angle $72^{\circ}$ (shown in red). The location of the galactic center is shown according to our convention $\left(\mathrm{PA}=26^{\circ}\right)$ and that of van der Marel (2001) $\left(\mathrm{PA}=42^{\circ}\right.$, shown in green). The direction of motion of the LMC is in the same quadrant as the line connecting the LMC and the GC, which is the northeast quadrant. The location of the SMC is such that the LMC lies in the line of interaction of the SMC and the MW. This line is also in the northeast direction. It is quite possible that the recent star-formation in the Clouds is due to the effect of the gravitational attraction of the MW on the HI gas within them.

We propose the following scenario to explain the lopsidedness in the LMC. We suggest that the HI gas in the LMC is shifted/pulled to the north by the perigalactic passage at about 200 Myr. The gas in the north is shocked/compressed to form stars starting from about $200 \mathrm{Myr}$, owing to the motion of the LMC in the MW halo. The efficiency in converting gas to stars seems to have increased in the 100-40 Myr age range. The direction of the center shift is more or less in the direction of the line connecting the LMC and the MW. At about $40 \mathrm{Myr}$, we detect the northeastern regions to have enhanced star-formation, suggesting that there has been efficient compression/shocking in the northeast. The LMC is moving away from the MW, after their closest approach. This could result in the northeast becoming more compressed than the north, which is the direction of motion of the LMC. We do not detect any age difference or propagating star-formation along the northern blue arm. The northeast enhancement in star-formation is probably due to the compression of gas by the motion of the LMC. A similar scenario of bowshock induced star-formation was proposed by de Boer et al. (1998). They suggested that the gas gets compressed in the east and then moves towards north with the LMC disk rotation. This implies that there should be a gradient in the age along the northern region from the east, but such a gradient is not seen here. We suggest that this compression has been active only in the last $40 \mathrm{Myr}$ and not before then.

Another result of this study is the detection of an outside-toinside quenching of star-formation within the last $100 \mathrm{Myr}$. We identify a peak in the star-formation at 90-100 Myr, which was also identified by H\&Z09. The peak of star-formation found at $100 \mathrm{Myr}$ is probably a global feature in the LMC, which is probably the effect of the perigalactic passage on the HI gas of the LMC. This star-formation was present in most of the regions and the star-formation then became restricted to the inner LMC. Thus, the star-formation was quenched from the outside to the inside after the 100 Myr peak. In the central regions, there is an indication that most of the regions stopped forming stars in 30$40 \mathrm{Myr}$ age range with only a few pockets continuing to form stars. On the other hand, the northeast regions, southwest end of the bar, and some northern regions continued to form stars until very recently, giving rise to the $0-10 \mathrm{Myr}$ peak in starformation. The quenching of star-formation was efficient in the southern LMC as most of the gas present was converted to stars and there is not much gas left. The star-formation in the north and the northeast continues owing to the presence of gas and the efficient compression produced by the motion of the LMC.

The case of the SMC has been found to be different. We have detected peaks of star-formation at 0-10 Myr and at 50-60 Myr. The recent star-formation is not found to be as structured as in the LMC. We have detected a shift in the center of the stellar population younger than $500 \mathrm{Myr}$. This might be due to the interaction of the SMC with the LMC about 400 Myr ago, as suggested by the coincident star-formation (H\&Z09). We have detected an enhanced shift in the center of the population in the 200-40 Myr age range, which is similar to that found in the LMC. The directions of the lines connecting the LMC and the Galaxy to the SMC, as shown in Fig. 25, are similar. This means that the gravitational effect of the LMC and the Galaxy on the SMC will be in the similar direction. Thus, shift in the center of the stars in the 200-40 Myr age range may be a combined effect of the LMC as well as the perigalactic passage. In the SMC, we also see that the northeastern regions are active in star-formation, relative to the south and the west. This may also be due to the effective compression of the HI gas in the northeastern regions of the $\mathrm{SMC}$, owing in turn to the motion of the LMC-SMC system in the halo of the MW.

In summary, the recent star-formation in the LMC has been dictated by the last perigalactic passage. The timescales and locations of star-formation identified in this study are valuable to model the recent interactions between the Clouds and the MW. The lopsidedness of the HI distribution towards the north can provide constraints on the parameters governing the gravitational force of the Galaxy on the LMC, before, during, and after the perigalactic passage. The compression of gas in the northern regions during the perigalactic passage and the compression of gas in the last 40 Myr can also place constraints on the direction of motion of the LMC, as well as the effect of the Galactic halo on the HI gas. It will be interesting to generate these details in the LMC disk, using an SPH simulation. The recent star-formation in the SMC is complicated because of the combined gravitational 
Table A.1. The input parameters to the synthetic CMD and the estimated LSFE, $A_{v}$ values in the L\&SMC.

\begin{tabular}{cccccc}
\hline \hline & $\log ($ age $)$ & nstar\# & Mass $\left(M_{\odot}\right)$ & LSFE log $($ age $)$ & Derived $A_{v}$ \\
\hline LMC & 7.40 & 500 & 1124.5 & 7.88 & 0.7018 \\
& & 1000 & 2361.3 & 7.90 & 0.4538 \\
& & 1500 & 3670.9 & 7.49 & 0.5878 \\
& & 2000 & 4980.1 & 7.34 & 0.5878 \\
& & 2500 & 6258.8 & 7.41 & 0.5878 \\
& & 3000 & 7546.2 & 7.41 & 0.5878 \\
& & 3500 & 8824.9 & 7.41 & 0.5878 \\
SMC & 4.50 & 5000 & 10133.7 & 7.41 & 0.5878 \\
& & 1123.1 & 8.11 & 0.4290 \\
& & 1000 & 2372.7 & 7.95 & 0.4712 \\
& & 2000 & 3666.4 & 7.62 & 0.5332 \\
& & 4987.3 & 7.54 & 0.5630 \\
& 3000 & 6299.9 & 7.54 & 0.5630 \\
& 3500 & 8990.9 & 7.54 & 0.5630 \\
& 4000 & 10392.2 & 7.54 & 0.5630 \\
& & & & & 0.5630 \\
\hline
\end{tabular}

Notes. The extinction applied for synthesising CMD are 0.55 for the LMC and 0.46 for the SMC.

effect of the LMC and the Galaxy, especially with both of them located in the same direction. Thus, disentangling the effect on the SMC caused by its interaction with the LMC and the effect of the perigalactic passage may be difficult.

Acknowledgements. We are grateful to the referee Dr. Antonio Aparicio for his valuable remarks and suggestions, which improved the presentation of the results of the paper. We gratefully acknowledge Dr. Eva K. Grebel and Dr. Gurtina Besla for their comments on the paper. We thank Katharina Glatt for providing the star cluster data and Dr. Snezana Stanimirovic for providing the HI data in the SMC. Thanks to Smitha Subramanian for interesting discussions and Dr. Armando Ferro, Dr. Stalin and Dr. Firoza Sutaria for valuable inputs.

\section{Appendix A: Error analysis}

In this study, we used a simple method of identifying the MS turn-off and converting into the age of the youngest starformation. The ages estimated by this method, are in general, found to be comparable with the cluster ages and ages estimated by H\&Z09. The ages estimated here are affected by the photometric errors, errors in the estimation of extinction, and the finite resolution in binning along the luminosity as well as the colour axes. We derived the error in the estimated values of extinction and LSFE age using two methods: (1) synthetic CMDs and (2) the propagation of errors. These are described below.

We synthesised CMDs with an IMF 2.35, in the mass range 0.6-20 $M_{\odot}$, incorporating the observed (typical) photometric error and using the Marigo et al. (2008) isochrones for metallicities 0.008 (LMC) and 0.004 (SMC). Synthetic CMDs are created for turn-off ages in the range of 10 to $100 \mathrm{Myr}$. To quantify the sampling effects and errors in the derived turn-off age, we performed the same analysis on the synthesised data. For each population, we estimated the LSFE age and extinction. To understand the effect of sampling, the analysis was repeated by varying the total number of stars in a CMD. We applied an $A_{v}$ of $0.55 \mathrm{mag}$ to the LMC and $0.46 \mathrm{mag}$ for the SMC to synthesise the CMDs. The LSFE age and extinction estimated from the synthetic CMDs were compared with the input values to find the error. To incorporate the effects of sampling, the number of stars (or total mass) is also varied. Synthetic CMDs are created with a wide range in the number of stars (more as well as less than that found typically in the observed CMDs). As an example, the
Table A.2. The error in the derived ages estimated using synthesised data.

\begin{tabular}{cccc}
\hline \hline & $\log ($ age $)$ & Error in log $($ age $)$ & Mass $\left(M_{\odot}\right)$ \\
\hline LMC & 7.0 & 0.15 & 7550 \\
& 7.10 & 0.15 & 4980 \\
& 7.35 & 0.10 & 4274 \\
& 7.40 & 0.09 & 3671 \\
& 7.50 & 0.07 & 4986 \\
& 7.60 & 0.06 & 3668 \\
& 7.70 & 0.06 & 4280 \\
& 7.75 & 0.04 & 3657 \\
& 7.90 & 0.02 & 2367 \\
& 8.00 & 0.03 & 2362 \\
\hline SMC & 7.0 & 0.15 & 6298 \\
& 7.2 & 0.17 & 6310 \\
& 7.3 & 0.15 & 4975 \\
& 7.4 & 0.14 & 3680 \\
& 7.5 & 0.12 & 3666 \\
& 7.55 & 0.11 & 3669 \\
& 7.65 & 0.12 & 3680 \\
& 7.70 & 0.07 & 3669 \\
& 7.80 & 0.07 & 2356 \\
& 7.90 & 0.05 & 2375 \\
& 8.00 & 0.02 & 2366 \\
\hline
\end{tabular}

Notes. The tabulated error holds for synthetic CMDs with minimum mass shown in Col. 4.

input parameters for the synthetic CMD and the estimated results for the L\&SMC for a single age are tabulated in Table A.1. Columns 1-3 are the log (age), the total number of stars and the total stellar mass in the synthetic CMD. The total stellar mass can be used to estimate the typical star-formation rate, which is detected using this method (and the cut-off) as a function of age. The star-formation rate can be estimated by dividing the total mass with the age range corresponding to the $M_{v}$ and the bin size. Columns 4 and 5 are the estimated LSFE log (age) and extinction. The analysis was performed for various age ranges and the results are tabulated in Table A.2. The columns are (log) age, estimated error in age, and the minimum mass (and hence the number of stars) required to produce the synthetic CMD, with the tabulated parameter. This number is found to be in the range 1500-2000 and is smaller than the number of stars found in the observed CMDs above the limiting magnitude. The maximum error in the extinction is found to be within 2.48 times the bin size $(0.1 \mathrm{mag})$ of the $(V-I)$ colour. The error in log (age) is found to be in the range of $0.01-0.15$ in the case of the LMC and $0.01-0.17$ in the case of the SMC. This error includes the error in the estimation of extinction and magnitude of the MS turn-off, the statistical error, and a typical photometric error. This method gives an estimate in the error as a function of the LSFE age.

The error in the estimated LSFE as a function of location is estimated using the second method. The error is calculated by means of the propagation of error method, starting with the photometric error in $V$ and $I$ bands, $\sigma V$ and $\sigma(V-I)$.

$$
\begin{aligned}
\sigma A_{v} & =2.48 \sqrt{\sigma(V-I)^{2}+(V-I)_{\mathrm{bin}}^{2}}, \\
\sigma M_{v} & =\sqrt{\sigma V^{2}+V_{\mathrm{bin}}^{2}+\sigma A_{v}^{2}}, \\
\sigma \mathrm{age} & =\text { const. } \times \sigma M_{v},
\end{aligned}
$$

where $V_{\text {bin }}$ and $(V-I)_{\text {bin }}$ are half the bin sizes used for magnitude and color binning, $\sigma M_{v}$ is the error in absolute magnitude, $\sigma A_{v}$ is the error in the estimated extinction and $\sigma$ age is the error in LSFE age on a logarithmic scale. The spatial plots of the 

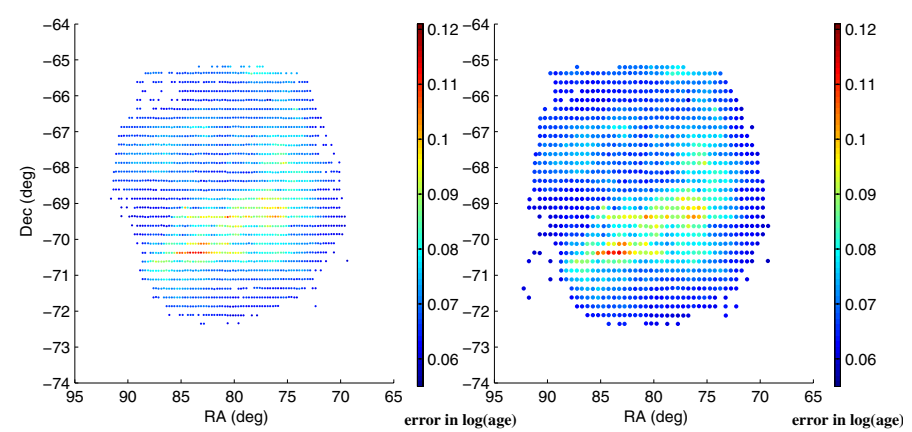

(A)

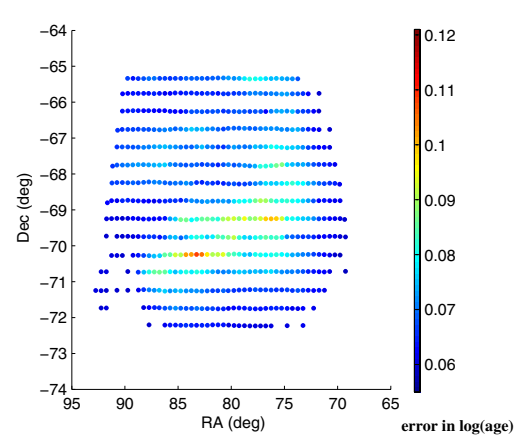

Error map - LMC (MCPS)

Bin size used

A) $5.3 \times 15 \operatorname{arcmin}^{2}$

B) $10.5 \times 15 \operatorname{arcmin}^{2}$

C) $10.5 \times 30 \operatorname{arcmin}^{2}$

Fig. A.1. The error in estimated age for the LMC using MCPS data for all the three area bins as indicated in the figure. Color coding is according to the error in log (age).

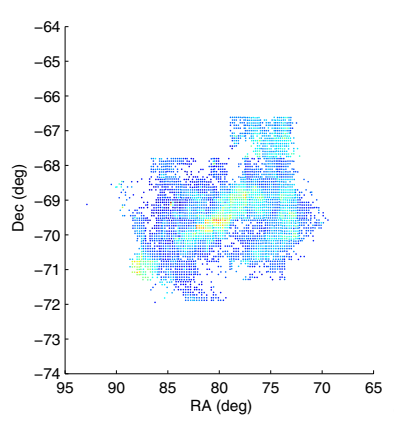

(A)

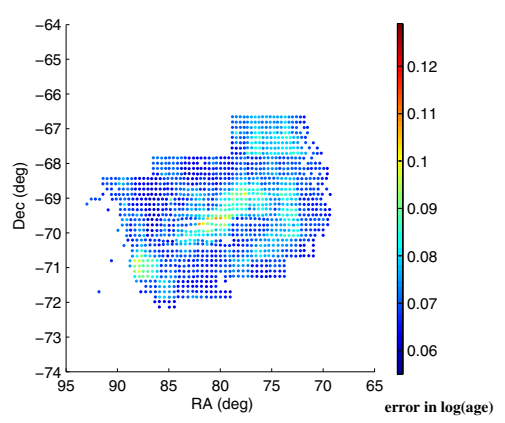

(C)

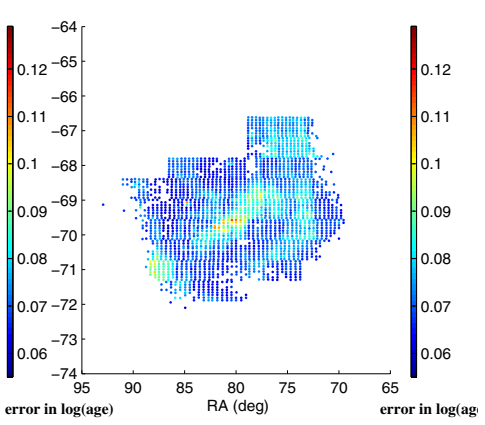

error map - LMC (OGLE III)

Bin size used

A) $4.4 \times 4.4 \operatorname{arcmin}^{2}$

B) $4.4 \times 8.8 \operatorname{arcmin}^{2}$

C) $8.8 \times 8.8 \operatorname{arcmin}^{2}$

Fig. A.2. Error map for the LMC using OGLE III, similar to Fig. A.1.

errors are shown in Figs. A.1 to A.4, for L\&SMC for both the data sets MCPS and OGLE III. The error in log (age) varies from 0.06 to 0.12 . The error is found to be higher along the bar of the LMC and the central regions of SMC. This is primarily the effect of photometric errors due to the crowding, as these are the densest regions in the respective galaxies. We identify a larger error for the OGLE III data than forthe MCPS data for the central

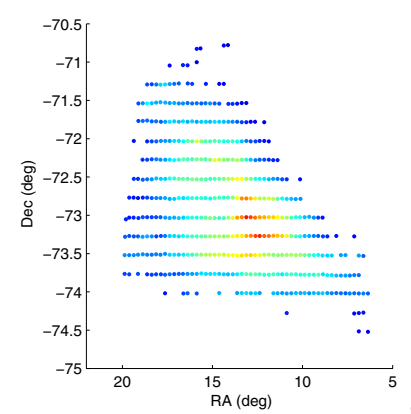

(A)

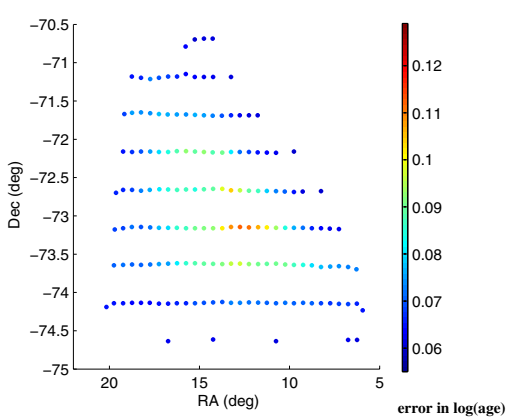

(C)

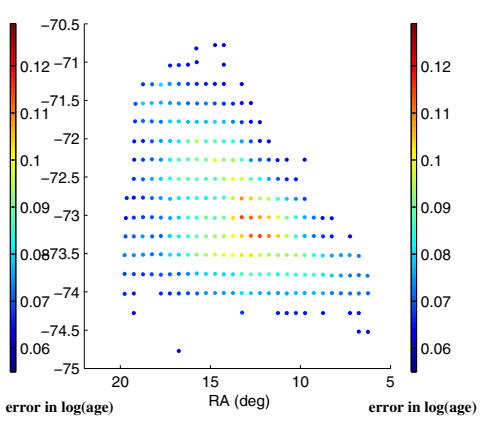

(B)

Error map - SMC (MCPS)

Bin size used

A) $5.3 \times 15 \operatorname{arcmin}^{2}$

B) $10.5 \times 15 \operatorname{arcmin}^{2}$

C) $10.5 \times 30 \operatorname{arcmin}^{2}$
Fig. A.3. Error in age estimation shown as a map for the SMC using MCPS data for all the three area bins as indicated in the figure. Color coding is according to the error in log (age).

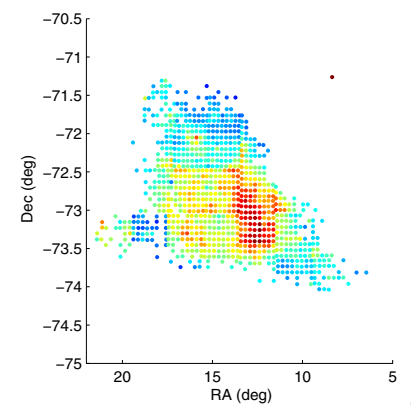

(A)

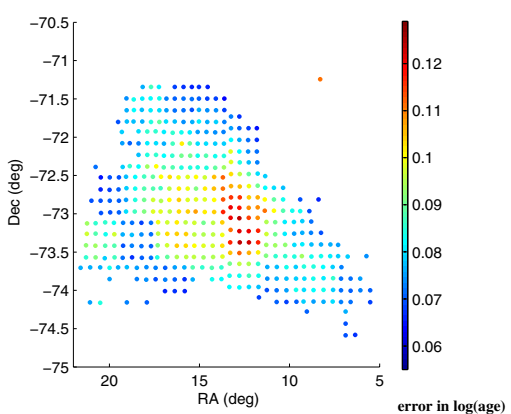

(C)

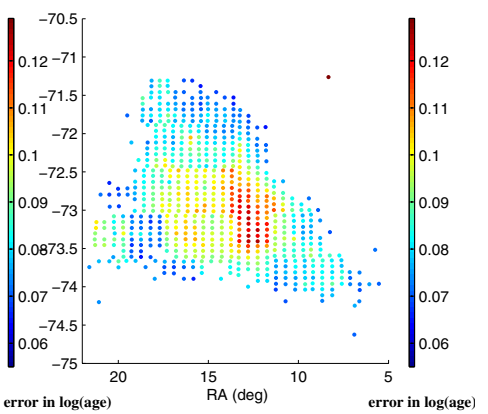

(B)
Bin size used

A) $4.4 \times 4.4 \operatorname{arcmin}^{2}$

B) $4.4 \times 8.8 \mathrm{arcmin}^{2}$

C) $8.8 \times 8.8 \operatorname{arcmin}^{2}$

Fig. A.4. Error map for the SMC using OGLE III, similar to Fig. A.3.

regions of the SMC. The error in the LSFE age, as estimated by the above two methods are comparable. The first method is likely to estimate the true error and the second method is likely to overestimate the error. Both the methods are included to show the variation of error as a function of both age and location. 


\section{References}

Alcock, C., \& Allsman, R. A. 1999, ApJ, 117, 920

Bekki, K., \& Chiba, M. 2009, PASA, 29, 48

Besla, G., \& Kallivayalil, N. 2007, ApJ, 668, 949

Besla, G., Kallivayalil, N., \& Hernquist, L. 2010, ApJ, 721, 97

Bica, E., Claria, J. J., \& Dottori, H. 1992, AJ, 103, 1859

de Boer, K. S., Braun, J. M., Vallenary, A., \& Mebold, U. 1998, A\&A, 329, 49

de Vaucouleurs, G., \& Freeman, K. C. 1973, Vistas Astron., 14, 163

Dottori, H., \& Bica, E. 1996, ApJ, 461, 742

Gallart, C., Stetson, P. B., Meschin, P. I., Pont, F., \& Hardy, E. 2008, AJ, 682,

L89

Gallart, C., Meschin, P. I., Aparicio, A., Stetson, P. B., \& Hidalgo, S. L. 2009, IAUS, 256, 281

Glatt, K., Grebel, E. K., \& Koch, A. 2010, A\&A, 517, 50

Harris, J., \& Zaritsky D. 2004, AJ, 127, 1531

Harris, J., \& Zaritsky D. 2009, ApJ, 138, 1243

Holtzman, J. A., Gallagher, J. S., Cole, A. A., et al. 1999, AJ, 118, 2262

Kim, S., Staveley-Smith, L., Dopita, M. A., et al. 1998, ApJ, 503, 674

Kim, S., Dopita, M. A., Staveley-Smith, L., \& Bessell, M. 1999, AJ, 118, 2797

Kim, S., Rosolowsky, E., \& Lee, Y. 2007, ApJS, 171, 419

Lin, D. N. C., Jones, B. F., \& Klemola, A. R. 1995, ApJ, 439, 652

Marigo, P., Girardi, L., Bressan, A., et al. 2008, A\&A 482, 883

Meaburn, J. 1980, MNRAS, 192, 365
Nikolaev, S., Drake, A. J., Keller, S. C., et al. 2004, ApJ, 601, 260

Noel, N. E. D., Aparicio, A., Gallart, C., et al. 2009, ApJ, 705, 1260

Piatek, S., Pryor, C., \& Olszewski, E. W. 2008, AJ, 135, 1024

Pietrzynski, G., \& Udalski, A. 2000, A\&A, 50, 337

Saha, A., Olszewski, E. W., Brondel, B., et al. 2010, AJ, 140, 1719

Stanimirovic, S., Staveley-Smith, L., Dickey, J. M., et al. 1999, MNRAS, 302. 417

Stanimirovic, S., Staveley-Smith, L., \& Jones, P. A. 2004, ApJ, 604, 176, 186 Subramaniam, A. 2004, A\&A, 425, 837

Udalski, A., Soszynski, I., Szymanski, M., et al. 2008a, Acta Astron., 58, 89 (LMC OGLE III data)

Udalski, A., Soszynski, I., Szymanski, M., et al. 2008b, Acta Astron., 58, 329 (SMC OGLE III data)

van der Marel, R. P. 2001, AJ, 122, 1827

van der Marel, R. P., \& Cioni, M. L. 2001, AJ, 122, 1807

Vieira, K., Girard, M., T., van Altena, F., et al. 2010, AJ, 140, 1934

Weinberg, M. D., \& Nikolaev, S. 2001, ApJ, 548, 712

Westerlund, B. E., 1997, The Magellanic Clouds (Cambridge: Cambridge Univ. Press)

Zaritsky, D., Harris, J., Thompson, I. B., et al. 2002, AJ, 123, 855 (SMC MCPS data)

Zaritsky, D., Harris, J., Thompson, I. B., et al. 2004, AJ, 128, 1606 (LMC MCPS data) 